\title{
Gaussian process optimization with failures: classification and convergence proof
}

\author{
F. Bachoc ${ }^{1}$, C. Helbert ${ }^{2}$, V. Picheny ${ }^{3}$ \\ ${ }^{1}$ Institut de Mathématiques de Toulouse, Université Paul Sabatier \\ ${ }^{2}$ Univ. de Lyon, Ecole Centrale de Lyon, CNRS UMR 5208, \\ Institut Camille Jordan, 36 av. G. de Collongue F-69134 Ecully cedex, FRANCE \\ ${ }^{3}$ PROWLER.io, Cambridge, UK
}

January 29,2020

\begin{abstract}
We consider the optimization of a computer model where each simulation either fails or returns a valid output performance. We first propose a new joint Gaussian process model for classification of the inputs (computation failure or success) and for regression of the performance function. We provide results that allow for a computationally efficient maximum likelihood estimation of the covariance parameters, with a stochastic approximation of the likelihood gradient. We then extend the classical improvement criterion to our setting of joint classification and regression. We provide an efficient computation procedure for the extended criterion and its gradient. We prove the almost sure convergence of the global optimization algorithm following from this extended criterion. We also study the practical performances of this algorithm, both on simulated data and on a real computer model in the context of automotive fan design.
\end{abstract}

\section{Introduction}

Bayesian optimization (BO) is now established as an efficient tool for solving optimization problems with non-linear objectives that are expensive to evaluate. A wide range of applications have been tackled, from hyperparameter tuning of machine learning algorithms [31] to wing shape design [16]. In the simplest BO setting, the aim is to find the maximum of a fixed unknown function $f: \mathcal{D} \rightarrow \mathbb{R}$, where $\mathcal{D}$ is a box of dimension $d$. Under that configuration, the classical Efficient Global Optimization [EGO, 13] and its underlying acquisition function Expected Improvement (EI) are still considered state-of-the-art.

Several authors have adapted BO to the constrained optimization framework, i.e. when the acceptable design space $\mathcal{A} \subset \mathcal{D}$ is defined by a set of non-linear, expensive-to-compute equations $c$ :

$$
\mathcal{A}=\{x \in \mathcal{D} \text { s.t. } c(x) \leq 0\},
$$

either by adapting the EI function [30, 29, 6, 11, 25] or by proposing alternative acquisition functions [24, 12].

We consider here the problem of crash constraints, where the objective $f$ is typically evaluated using a computer code that fails to provide simulation results $f(x)$ for some input 
conditions $x$. We write $\mathcal{A}$ of the form

$$
\mathcal{A}=\{x \in \mathcal{D} ; s(x)=1\}
$$

where $s: \mathcal{D} \rightarrow\{0,1\}$ is a fixed unknown function.

We assume that, for each $x \in \mathcal{D}$, a single computation provides the pair $\left(s(x), \mathbf{1}_{s(x)=1} f(x)\right)$. Hence, it is as costly to see if a simulation at $x$ fails as to observe the simulation result $f(x)$ when there is no failure. A typical example of failure might be a computational fluid dynamics (CFD) solver that does not converge. This convergence failure could be caused by an overly large time-step yielding an instability in the numerical scheme and a divergence, or by an inadequate mesh close to the boundary of the domain (see also the discussions in [28]). Another typical example of failure is when $f(x)$ provides the numerical performance (e.g. the empirical risk) of a complex machine learning model (e.g. a deep neural network) depending on architecture parameters in $x$ [15]. The computation of $f(x)$ then relies on a gradient or stochastic gradient descent, using retro-propagation in the case of deep learning, for example. In this case, a failure occurs when the gradient descent does not converge, so that there is no observable value of $f(x)$ at convergence. In these two examples, we note that it is no less costly to observe a failure of the form $s\left(x_{1}\right)=0$ than to successfully observe $f\left(x_{2}\right)$ with $s\left(x_{2}\right)=1$.

This optimization problem with failures was considered first by [10], where a Gaussian process classifier [GPC, 22] was used together with a spatialized EI. [17] also proposed the use of a GPC with EI, modified using an asymmetric entropy to limit as much as possible the computational resources spent on crashed simulations. However, both approaches rely on expensive Monte Carlo simulations, which make them impractical in some cases, and do not provide any convergence guarantee.

The contribution of this paper is two-fold. First, a new GPC model is proposed, where a latent GP is simply conditioned on the signs of the observations instead of their values. Its likelihood function maximization is studied, as well as its use to predict the feasibility probability (i.e. crash likeliness) of a new design $x$. Second, leveraging recent results on sequential strategies [2], we propose an algorithm in the form of EGO with guaranteed convergence.

The outline of this paper is as follows. First, we introduce our GPC model (Section 2) and its use in a Bayesian optimization algorithm (Section 3). Section 4 states our main consistency result. Finally, our algorithm is illustrated on several simulated toy problems (Section 5), and applied to an industrial case study (Section 6). A conclusion is given in Section 7, All the proofs are deferred to the appendix.

\section{A Classification model for crash constraints}

This section presents our classification model used to characterize the feasible space $\mathcal{A}$. It takes the classical form of a GPC with a latent GP, but conditioned solely on pointwise observations of its sign.

\subsection{Conditioning GPs on observation signs}

Let $Z$ be a Gaussian process on $\mathcal{D}$ that has a constant mean function with value $\mu^{Z} \in \mathbb{R}$ and stationary covariance function $k^{Z}$. Given a set of points $x_{1}, \ldots, x_{n} \in \mathcal{D}$ and corresponding 
observations $Z_{n}=\left(Z\left(x_{1}\right), \ldots, Z\left(x_{n}\right)\right)^{\top}$, GP regression typically amounts to using the posterior mean $m_{n}^{Z}\left(x, z_{n}\right)=\mathbb{E}\left(Z(x) \mid Z_{n}=z_{n}\right)$ and variance $k_{n}^{Z}(x)=\operatorname{Var}\left(Z(x) \mid Z_{n}=z_{n}\right)$, for $z_{n} \in \mathbb{R}^{n}$.

Now, in the classification setting, $Z$ is a latent process and $Z_{n}$ is not available. We propose here to predict $\mathbf{1}_{Z(x)>0}$ given the sign of $Z_{n}$; that is, we consider the conditional non-failure probability

$$
\mathrm{P}_{\mathrm{nf}}(x)=\mathbb{P}\left(Z(x)>0 \mid \operatorname{sign}\left(Z_{n}\right)=s_{n}\right),
$$

where $s_{n}=\left(i_{1}, \ldots, i_{n}\right)^{\top}$ with $i_{1}, \ldots, i_{n} \in\{0,1\}$ and $\operatorname{sign}(v)=\left(\mathbf{1}_{v_{1}>0}, \ldots, \mathbf{1}_{v_{n}>0}\right)^{\top}$ for $v=$ $\left(v_{1}, \ldots, v_{n}\right) \in \mathbb{R}^{n}$.

To our knowledge, there is no exact integral-free expression of $\mathrm{P}_{\mathrm{nf}}(x)$. The following lemma provides an expression of $\mathrm{P}_{\mathrm{nf}}(x)$ that is more amenable to numerical approximation.

Lemma 1. For $s_{n} \in\{0,1\}^{n}$, let $\phi_{s_{n}}^{Z_{n}}$ be the conditional p.d.f. of $Z_{n}$ given $\operatorname{sign}\left(Z_{n}\right)=s_{n}$. Let us define, for $a \in \mathbb{R}, b \geq 0$,

$$
\bar{\Phi}\left(\frac{a}{b}\right)=\left\{\begin{array}{ll}
1-\Phi\left(\frac{a}{b}\right) & \text { if } b \neq 0 \\
\mathbf{1}_{-a>0} & \text { if } b=0
\end{array},\right.
$$

where $\Phi$ is the standard Gaussian c.d.f. Then we have

$$
\mathrm{P}_{\mathrm{nf}}(x)=\int_{\mathbb{R}^{n}} \phi_{s_{n}}^{Z_{n}}\left(z_{n}\right) \bar{\Phi}\left(\frac{-m_{n}^{Z}\left(x, z_{n}\right)}{\sqrt{k_{n}^{Z}(x)}}\right) d z_{n} .
$$

Proof. The proof is deferred to Appendix A.

Because of Lemma 1, we suggest the following algorithm to approximate $\mathrm{P}_{\mathrm{nf}}(x)$.

\section{Algorithm 1.}

1. Sample $z_{n}^{(1)}, \ldots, z_{n}^{(N)} \in \mathbb{R}^{n}$ from the p.d.f. $\phi_{s_{n}}^{Z_{n}}$.

2. For any $x \in \mathcal{D}$, approximate $\mathrm{P}_{\mathrm{nf}}(x)$ by

$$
\widehat{\mathrm{P}_{\mathrm{nf}}}(x)=\frac{1}{N} \sum_{i=1}^{N} \bar{\Phi}\left(\frac{-m_{n}^{Z}\left(x, z_{n}^{(i)}\right)}{\sqrt{k_{n}^{Z}(x)}}\right) .
$$

The benefit of Algorithm 1 is that Step 1, which is the most costly, has to be performed only once (independently of $x \in \mathcal{D}$ ). In this step, $z_{n}^{(1)}, \ldots, z_{n}^{(N)}$ can be sampled either by a basic rejection method (sampling $Z_{n}$ from its Gaussian p.d.f. $\phi^{Z_{n}}$ until the signs of $Z_{n}$ match $\left.i_{1}, \ldots, i_{n}\right)$, or by a more advanced rejection method called Rejection Sampling from the Mode (RSM) [19], or by more involved Markov Chain Monte Carlo (MCMC) methods [4, 33, 23]; see also their presentations in [18. Step 2 is not costly and can be repeated for many inputs $x$. 


\subsection{Likelihood computation and optimization}

Let $\left\{k_{\theta}^{Z} ; \theta \in \Theta\right\}$ be a set of stationary covariance functions on $\mathcal{D}$ with $\Theta \subset \mathbb{R}^{p}$. Typically, $\theta$ consists of an amplitude term and one or several lengthscale terms [26, 27]. We aim at selecting a constant mean function for $Z$ with value $\mu \in \mathbb{R}$ and a covariance parameter $\theta$. Let us first consider two pairs $\left(\theta_{1}, \mu_{1}\right),\left(\theta_{2}, \mu_{2}\right) \in \Theta \times \mathbb{R}$ for which $k_{\theta_{1}}^{Z} / k_{\theta_{1}}^{Z}(0)=k_{\theta_{2}}^{Z} / k_{\theta_{2}}^{Z}(0)$ and $\mu_{1} /\left(k_{\theta_{1}}^{Z}(0)\right)^{1 / 2}=\mu_{2} /\left(k_{\theta_{2}}^{Z}(0)\right)^{1 / 2}$. Then, we can check that the distribution of the sign process $\left\{1_{Z(x)>0} ; x \in \mathcal{D}\right\}$ is the same when $Z$ has mean and covariance function $\mu_{1}$ and $k_{\theta_{1}}$ or $\mu_{2}$ and $k_{\theta_{2}}$. Hence, it is sufficient to let $\left\{k_{\theta}^{Z} ; \theta \in \Theta\right\}$ be a set of stationary correlation function and to let $\mu \in \mathbb{R}$ be unrestricted.

For $s_{n} \in\{0,1\}^{n}$, let $\mathbb{P}_{\mu, \theta}\left(\operatorname{sign}\left(Z_{n}\right)=s_{n}\right)$ be the probability that $\operatorname{sign}\left(Z_{n}\right)=s_{n}$, calculated when $Z$ has mean function $\mu$ and covariance function $k_{\theta}$. Then, the maximum likelihood estimators for $\mu$ and $\theta$ are

$$
(\hat{\mu}, \hat{\theta}) \in \underset{(\mu, \theta) \in \mathbb{R} \times \Theta}{\operatorname{argmax}} \mathbb{P}_{\mu, \theta}\left(\operatorname{sign}\left(Z_{n}\right)=s_{n}\right) .
$$

The likelihood criterion to optimize is the probability of an orthant of $\mathbb{R}^{n}$, evaluated under a multidimensional Gaussian distribution. Several advanced Monte Carlo methods exist to approximate this probability [4, 7, 1]. In addition, stochastic approximations of the gradient of $\mathbb{P}_{\mu, \theta}\left(\operatorname{sign}\left(Z_{n}\right)=s_{n}\right)$ with respect to $(\mu, \theta)$ can be obtained from conditional realizations of $Z_{n}$ given $\operatorname{sign}\left(Z_{n}\right)=s_{n}$. Calculations are provided in Appendix B.

\subsection{Comparison with classical GPC}

The model in Sections 2.1 and 2.2 can be written as

$$
I_{i}=\mathbf{1}_{Z\left(x_{i}\right)>0} \text { for } i=1, \ldots, n \text { and } I=\mathbf{1}_{Z(x)>0},
$$

where $I_{1}, \ldots, I_{n} \in\{0,1\}$ are observed and $I \in\{0,1\}$ is to be predicted. In the model (2), the parameters to estimate are the constant mean $\mu \in \mathbb{R}$ and the correlation parameter $\theta$ for $Z$.

Another widely used Gaussian process-based classification model is the one given in [26, 22. In this model, there is again a Gaussian process $\mathrm{Z}$ and, conditionally on $Z\left(x_{1}\right), \ldots, Z\left(x_{n}\right), Z(x)$, the variables $I_{1}, \ldots, I_{n}, I$ are independent and take values 0 or 1 . Furthermore, with $Z_{n}=$ $\left(Z\left(x_{1}\right), \ldots, Z\left(x_{n}\right)\right)^{\top}$ again:

$$
P\left(I_{i}=1 \mid Z_{n}, Z(x)\right)=\operatorname{sig}\left(\sigma_{f} Z\left(x_{i}\right)\right) \text { for } i=1, \ldots, n \text { and } P\left(I=1 \mid Z_{n}, Z(x)\right)=\operatorname{sig}\left(\sigma_{f} Z(x)\right),
$$

where sig $: \mathbb{R} \rightarrow(0,1)$ is a continuous strictly increasing function satisfying $\operatorname{sig}(t) \rightarrow 0$ as $t \rightarrow-\infty$ and $\operatorname{sig}(t) \rightarrow 1$ as $t \rightarrow+\infty$ and with $\sigma_{f}>0$. For instance, a classical choice in [26, 22] is the logit function defined by $\operatorname{sig}(t)=e^{t} /\left(1+e^{t}\right)$.

In the model (3), it is assumed in [26, 22] that the mean function of $Z$ is zerd ${ }^{1}$. The parameter to estimate for the covariance function of $Z$ is $\theta$, from the set of stationary covariance functions $\left\{k_{\theta} ; \theta \in \Theta\right\}$. The parameter $\sigma_{f}$ also has to be estimated. Since the mean function of $Z$ is assumed to be zero, one can see that pairs $\left(\theta_{1}, \sigma_{f, 1}\right)$ and $\left(\theta_{2}, \sigma_{f, 2}\right)$, for which $\sigma_{f, 1}^{2} k_{\theta_{1}}=\sigma_{f, 2}^{2} k_{\theta_{2}}$, give the same distribution of $I_{1}, \ldots, I_{n}, I$ in (3). Thus, for model (3), we let $\left\{k_{\theta} ; \theta \in \Theta\right\}$ be a set of correlation functions, and $\sigma_{f} \geq 0$ has to be estimated as well.

\footnotetext{
${ }^{1} \mathrm{~A}$ constant mean function could be incorporated and estimated with no additional complexity.
} 



Figure 1: GPC model (3) based on EP and the logit function (left) and our GPC model (2) (right).

We now compare our model (2) with (3). The framework (2) corresponds to the limit of the model in (3), as $\sigma_{f} \rightarrow+\infty$. Indeed, let $\operatorname{sgn}(t)=0$ if $t<0, \operatorname{sgn}(t)=1 / 2$ if $t=0$ and $\operatorname{sgn}(t)=1$ if $t>0$. Then, as observed in [22], when $\sigma_{f}=+\infty$, we have $P\left(I=1 \mid Z_{n}, Z(x)\right)=\operatorname{sgn}(Z(x))$ and $P\left(I_{i}=1 \mid Z_{n}, Z(x)\right)=\operatorname{sgn}\left(Z\left(x_{i}\right)\right)$, for $i=1, \ldots, n$. Since the components of $Z_{n}$ take values 0 with zero probability, (2) and (3) indeed give identical distributions of $\left(I_{1}, \ldots, I_{n}, I\right)$ when $\sigma_{f}=+\infty$.

In the framework described in Section 1, repeated calls to the code function for the same input $x$ either all crash or all successfully return an output value. Hence, model (2) is more appropriate than model (3) (especially with small values of $\sigma_{f}$ ). Figure 1 shows the two models built on a 50 point design of experiments (obtained from the uniform distribution) on a 2D toy problem. While model (3), based on expectation-propagation (EP) [22], returns a function with smooth transitions, our model (2) returns a much sharper function, which is more appropriate for a framework of deterministic failures. In addition, model (3) returns conditional crash probabilities that are not equal to exactly zero or one for input points with observed binary outputs. By contrast, the conditional probabilities returned by our model (2) are exactly zero or one for these input points with observed outputs. Again this is more appropriate for deterministic failures.

In terms of inference, we have discussed in Section 2.1 that, for a fixed $\theta$, the only costly step for model (2) is to sample realizations of the p.d.f. $\phi_{s_{n}}^{Z_{n}}$. This p.d.f. is that of a truncated Gaussian vector (restricted to an orthant of $\mathbb{R}^{n}$ ). Instead, the distribution to sample with model (3) (the conditional distribution of $Z_{n}$ given $I_{1}=i_{1}, \ldots, I_{n}=i_{n}$ ) admits a density on $\mathbb{R}^{n}$, which values at $z_{1}, \ldots, z_{n}$ are proportional to

$$
\left(\prod_{j=1}^{n} \operatorname{sig}\left(\sigma_{f} z_{j}\right)^{i_{j}}\left[1-\operatorname{sig}\left(\sigma_{f} z_{j}\right)\right]^{1-i_{j}}\right) \phi^{Z_{n}}\left(z_{1}, \ldots, z_{n}\right),
$$

where $\phi^{Z_{n}}$ is the Gaussian p.d.f. of $Z_{n}$. The density in (4) is arguably more complicated than a truncated Gaussian density function, for which many implemented algorithms are available, 
as discussed above when introducing the references [4, 33, 23, 18.

In [26, 22, several approximations of the distribution in (4) by multidimensional Gaussian distributions are presented (in particular, the Laplace and EP approximations, the variational method and the Kullback-Leibler method). These approximations are usually relatively fast to obtain from local optimization methods. Yet, they are approximations of a non-Gaussian distribution, and do not come (to our knowledge) with theoretical guarantees. Similarly, for parameter estimation, the likelihood function of $I_{1}, \ldots, I_{n}$ is approximated, and the approximation is maximized with respect to $\theta$ and $\sigma_{f}$. This yields a relatively fast procedure for estimating $\theta$ and $\sigma_{f}$, for which, again, no theoretical guarantees are available.

In contrast, with model (2), the simulation from the truncated conditional distribution $\phi_{s_{n}}^{Z_{n}}$, with $s_{n}=\left(i_{1}, \ldots, i_{n}\right)$ (Section 2.1) and the maximum likelihood estimation of $\theta$ and $\mu$ (Section 2.2) do not rely on approximations, and are based on Monte Carlo techniques rather than optimization. Hence, compared to model (3), the inference in model (2) may come with computational cost, but has more accuracy guarantees. For instance, there exists a large body of literature guaranteeing the convergence of Monte Carlo algorithms for long runs [20].

We assert that, with model (3) and the Gaussian approximation discussed above, once the conditional distribution of $\left(Z\left(x_{1}\right), \ldots, Z\left(x_{n}\right)\right)$ given $I_{1}=i_{1}, \ldots, I_{n}=i_{n}$ is approximated, it is not costly to obtain the conditional distribution of $I$ given $\left(I_{1}, \ldots, I_{n}\right)$ (see [26, 22]). This is similar to Algorithm 1 for model (2).

Finally, the constrained optimization problems addressed in the present article are of the form $\max _{x \in \mathcal{A}} f(x)$, where $\mathcal{A}$ is a fixed unknown subset. It is hence very natural to use the Bayesian prior $\{x \in \mathcal{D} ; Z(x)>0\}$ on $\mathcal{A}$, which is obtained from our classification model (2). In contrast, classification model (3) does not provide a fixed set of admissible inputs, since any $x$ in $\mathcal{D}$ has non-zero probabilities to yield both categories of the binary output. As a consequence, our suggested acquisition function in (9) below, and particularly its definition of the current admissible maximum $M_{q}$, rely on classification model (2). Hence, also the proof of convergence in Section 4 relies on classification model (2).

\section{Bayesian optimization with crash constraints}

Let us now address the case of optimization in the presence of computational failures. This problem requires a model for the objective function in addition to the one for the constraint. In this section, we first consider the problem of joint modeling, then its use in a Bayesian optimization algorithm.

\subsection{Joint modeling of the objective and constraint}

Let us consider two independent continuous Gaussian processes $Y$ and $Z$ from $\mathcal{D}$ to $\mathbb{R}$. In our framework, for an input point $x$, we can observe the pair

$$
(\operatorname{sign}[Z(x)], \operatorname{sign}[Z(x)] Y(x)) .
$$

That is, we observe whether the computation fails $(Z(x) \leq 0)$ or not, and in case of computation success, we observe the objective $Y(x)$.

For $Z$, as in Section 2, we select a constant mean $\mu^{Z} \in \mathbb{R}$ and a correlation parameter $\theta_{Z} \in \Theta_{Z}$, where $\left\{k_{\theta_{Z}}^{Z} ; \theta_{Z} \in \Theta_{Z}\right\}$ is a set of correlation functions with $\Theta_{Z} \subset \mathbb{R}^{p_{Z}}$. For $Y$, we 
select a constant mean $\mu^{Y} \in \mathbb{R}$ and a covariance parameter $\theta_{Y} \in \Theta_{Y}$, where $\left\{k_{\theta_{Y}}^{Y} ; \theta_{Y} \in \Theta_{Y}\right\}$ is a set of covariance functions with $\Theta_{Y} \subset \mathbb{R}^{p_{Y}}$.

Let the pair (5) be observed for the input points $x_{1}, \ldots, x_{n} \in \mathcal{D}$. For $j=1, \ldots, n$ we let $I_{j}=\operatorname{sign}\left(Z\left(x_{j}\right)\right)$ and consider the observation $\left(i_{1}, \ldots, i_{n}, i_{1} y_{1}, \ldots, i_{n} y_{n}\right)$ of

$$
\left(I_{1}, \ldots, I_{n}, I_{1} Y\left(x_{1}\right), \ldots, I_{n} Y\left(x_{n}\right)\right) .
$$

In the next lemma, we show that a likelihood can be defined for these $2 n$ observations. Since the distribution of $I_{i} Y\left(x_{i}\right)$ is a mixture of continuous and discrete distributions, we add a random continuous noise in case $I_{i}=0$. This random noise does not add or remove information, and is just a technicality in order to write the following lemma in terms of likelihood with respect to a simple fixed measure on $\mathbb{R}^{2 n}$.

Let us introduce some notation before stating the lemma. For $s_{n}=\left(i_{1}, \ldots, i_{n}\right)^{\top} \in$ $\{0,1\}^{n}$, let $Y_{n, s_{n}}$ be the vector extracted from $\left(Y\left(x_{1}\right), \ldots, Y\left(x_{n}\right)\right)$ by keeping only the indices $j \in\{1, \ldots, n\}$ for which $i_{j}=1$. Let $\phi_{\mu^{Y}, \theta_{Y}, s_{n}}$ be the p.d.f. of $Y_{n, s_{n}}$, calculated under the assumption that $Y$ has a constant mean function $\mu^{Y}$ and covariance function $k_{\theta_{Y}}^{Y}$. For $v=\left(v_{1}, \ldots, v_{n}\right)^{\top} \in \mathbb{R}^{n}$, let $v_{s_{n}}$ be the vector extracted from $v$ by keeping only the indices $j \in\{1, \ldots, n\}$ for which $i_{j}=1$.

Lemma 2. For $j=1, \ldots, n$, let $V_{j}=I_{j} Y\left(x_{j}\right)+\left(1-I_{j}\right) W_{j}$ where $W_{1}, \ldots, W_{n}$ are independent and follow the standard Gaussian distribution. Let $f_{\mu^{Z}, \theta_{Z}, \mu^{Y}, \theta_{Y}}$ be the p.d.f. of $\left(I_{1}, \ldots, I_{n}, V_{1}, \ldots, V_{n}\right)$, defined with respect to the measure $\left(\otimes_{i=1}^{n} \mu\right) \otimes\left(\otimes_{i=1}^{n} \lambda\right)$ where $\mu$ is the counting measure on $\{0,1\}$ and $\lambda$ is the Lebesgue measure on $\mathbb{R}$. Then we have

$$
\begin{aligned}
& f_{\mu^{Z}, \theta_{Z}, \mu^{Y}, \theta_{Y}}\left(i_{1}, \ldots, i_{n}, v_{1}, \ldots, v_{n}\right) \\
= & \mathbb{P}_{\mu^{Z}, \theta_{Z}}\left(I_{1}=i_{1}, \ldots, I_{n}=i_{n}\right) \phi_{\mu^{Y}, \theta_{Y}, s_{n}}^{Y}\left(v_{s_{n}}\right)\left(\prod_{\substack{j=1, \ldots, n \\
i_{j}=0}} \phi\left(v_{j}\right)\right),
\end{aligned}
$$

where $\phi$ is the standard Gaussian p.d.f. and $\mathbb{P}_{\mu^{z}, \theta_{Z}}(\cdot)$ is the probability of an event, calculated under the assumption that $Z$ has mean and covariance functions $\mu^{Z}$ and $k_{\theta_{Z}}^{Z}$.

Proof. The proof is deferred to Appendix A.

In view of Lemma 2, the maximum likelihood estimators of $\mu^{Z}, \theta_{Z}, \mu^{Y}, \theta_{Y}$ are

$$
\left(\hat{\mu}^{Z}, \hat{\theta}_{Z}\right) \in \underset{\left(\mu^{Z}, \theta_{Z}\right) \in \mathbb{R} \times \Theta_{Z}}{\operatorname{argmax}} \mathbb{P}_{\mu^{Z}, \theta_{Z}}\left(I_{1}=i_{1}, \ldots, I_{n}=i_{n}\right)
$$

and

$$
\left(\hat{\mu}^{Y}, \hat{\theta}_{Y}\right) \in \underset{\left(\mu^{Y}, \theta_{Y}\right) \in \mathbb{R} \times \Theta_{Y}}{\operatorname{argmax}} \phi_{\mu^{Y}, \theta_{Y}, s_{n}}^{Y}\left(Y_{q}\right),
$$

with $Y_{q}$ the realization of $Y_{n, s_{n}}$.

The likelihood maximization in (7) can be tackled as in Section 2, The likelihood maximization in (8) corresponds to the standard maximum likelihood in Gaussian process regression.

Once the likelihood has been optimized, it is common practice to take the optimal mean and covariance parameters at face value and neglect the uncertainty associated with their estimation (the "plugin" approach), although more Bayesian alternatives have been proposed [3], 
albeit at a higher computational cost. Note that, in practice, covariance parameters obtained from maximum likelihood estimation with data from deterministic functions can have undesirable properties in some cases. In particular, the estimates may depart substantially from oracle values (which would provide an efficient Gaussian process model for the deterministic function at hand) or even lead to failed runs in some cases [37]. In particular, overly large variance estimates may be obtained when working with the squared exponential covariance function [37. For this reason, it is important to study the covariance parameter estimates that are obtained carefully, which we do in the numerical examples in Section 5 . In addition, the squared exponential covariance function, leading to the potential issues described in [37, is not considered in Section 5 , the Matérn covariance functions are considered instead.

Under the plugin approach, we provide the conditional distributions of $Z$ and $Y$ in the following lemma, given the observations in (6).

Lemma 3. Conditionally on

$$
I_{1}=i_{1}, I_{1} Y\left(x_{1}\right)=i_{1} y_{1}, \ldots, I_{n}=i_{n}, I_{n} Y\left(x_{n}\right)=i_{n} y_{n}
$$

the stochastic processes $Y$ and $Z$ are independent. The stochastic process $Z$ follows the conditional distribution of $Z$ given $I_{1}=i_{1}, \ldots, I_{n}=i_{n}$ and the stochastic process $Y$ follows the conditional distribution of $Y$ given $Y_{n, s_{n}}=Y_{q}$, with $Y_{n, s_{n}}$ as in Lemma 2 and with $Y_{q}$ defined as in (8).

Proof. The proof is deferred to Appendix A,

In other words, conditionally on the observations, $Z$ is conditioned on its signs at $x_{1}, \ldots, x_{n}$, and $Y$ is conditioned on its values at the $x_{i}$ 's for which $Z\left(x_{i}\right)>0$. Hence, conditional inference on $Z$ can be carried out as described in Section 2, and $Y$ follows the standard Gaussian conditional distribution in Gaussian process regression.

\subsection{Acquisition function and sequential design}

Given the observations in (6), we now suggest an acquisition function that can be optimized to select a new observation point $x_{n+1} \in \mathcal{D}$, given a set of existing $n$ observations. We follow the classical improvement principle [21, 13, adapted to the partial observation setting. Thus, we choose:

$$
x_{n+1} \in \underset{x \in \mathcal{D}}{\operatorname{argmax}} \mathbb{E}\left(\mathbf{1}_{Z(x)>0}\left[Y(x)-M_{q}\right]^{+} \mid \mathcal{F}_{n}\right),
$$

where (with $\sigma(\cdot)$ the sigma-algebra generated by a set of random variables):

$$
\mathcal{F}_{n}=\sigma\left(I_{1}, I_{1} Y\left(x_{1}\right), \ldots, I_{n}, I_{n} Y\left(x_{n}\right)\right)
$$

denotes our observation event and:

$$
M_{q}=\max _{i=1, \ldots, n ; Z\left(x_{i}\right)>0} Y\left(x_{i}\right)
$$

with the convention $M_{q}=-\infty$ if $Z\left(x_{1}\right) \leq 0, \ldots, Z\left(x_{n}\right) \leq 0$. We call $\mathbb{E}\left(\mathbf{1}_{Z(x)>0}\left[Y(x)-M_{q}\right]^{+} \mid \mathcal{F}_{n}\right)$ the expected feasible improvement (EFI). 
As in Lemma 3, for $s_{n}=\left(i_{1}, \ldots, i_{n}\right) \in\{0,1\}^{n}$, we let $Y_{n, s_{n}}$ be the vector extracted from $\left(Y\left(x_{1}\right), \ldots, Y\left(x_{n}\right)\right)$ by keeping only the indices $j \in\{1, \ldots, n\}$ for which $i_{j}=1$. Thanks to this lemma we have:

$$
\begin{aligned}
\mathbb{E}\left(\mathbf{1}_{Z(x)>0}\left[Y(x)-M_{q}\right]^{+} \mid \mathcal{F}_{n}\right) & =\mathbb{P}\left(Z(x)>0 \mid I_{1}=i_{1}, \ldots, I_{n}=i_{n}\right) \mathbb{E}\left(\left[Y(x)-M_{q}\right]^{+} \mid Y_{n, s_{n}}\right) \\
& :=\mathrm{P}_{\mathrm{nf}}(x) \times \mathrm{EI}(x) .
\end{aligned}
$$

Hence, the EFI is equal to the product of the conditional probability of non-failure $\mathrm{P}_{\mathrm{nf}}(x)$ (conditionally on the signs of $Z$ ) and of the standard expected improvement $\operatorname{EI}(x)$ (conditionally on the observed values of $Y$ ). This criterion is similar to the one proposed in [30] and later [6] for quantifiable constraints. The criterion in [17] is slightly different in order to favor the exploration of the boundary, but at the loss of a consistent definition of improvement:

$$
\mathrm{EI}(x)^{\alpha_{1}} \times\left[\frac{2 \mathrm{P}_{\mathrm{nf}}(x)\left(1-\mathrm{P}_{\mathrm{nf}}(x)\right)}{\mathrm{P}_{\mathrm{nf}}(x)-2 w \mathrm{P}_{\mathrm{nf}}(x)+w^{2}}\right]^{\alpha_{2}},
$$

with $\alpha_{1}, \alpha_{1}$ and $w$ positive parameters.

The conditional probability of non-failure $\mathrm{P}_{\mathrm{nf}}(x)$ can be approximated by $\widehat{\mathrm{P}_{\mathrm{nf}}}(x)$ from Algorithm 1. In this algorithm, the first step is costly but needs to be performed only once independently of $x$, hence is outside the optimization loop (9). Then, $\widehat{\mathrm{P}_{\mathrm{nf}}}(x)$ is a smooth function of $x$ that is not costly to evaluate.

Turning to the expected improvement $\operatorname{EI}(x)$, let $q$ be the length of $Y_{n, s_{n}}$. For a realization $\left(y_{1}, \ldots, y_{n}\right)$ of $\left(Y\left(x_{1}\right), \ldots, Y\left(x_{n}\right)\right)$, let $Y_{q}$ be the vector extracted from $\left(y_{1}, \ldots, y_{n}\right)$ by keeping only the indices $j \in\{1, \ldots, n\}$ for which $i_{j}=1$. Hence, $Y_{q}$ is a realization of $Y_{n, s_{n}}$.

Let $x \rightarrow m_{q}^{Y}\left(x, Y_{q}\right)$ and $(x, y) \rightarrow k_{q}^{Y}(x, y)$ be the conditional mean and covariance functions of $Y$ given $Y_{n, s_{n}}=Y_{q}$. Let also $k_{q}^{Y}(x)=k_{q}^{Y}(x, x)$. It is well-known (see e.g.[13]) that

$$
\mathrm{EI}(x)=\left(m_{q}^{Y}\left(x, Y_{q}\right)-M_{q}\right) \Phi\left(\frac{m_{q}^{Y}\left(x, Y_{q}\right)-M_{q}}{\sqrt{k_{q}^{Y}(x)}}\right)+\sqrt{k_{q}^{Y}(x)} \phi\left(\frac{m_{q}^{Y}\left(x, Y_{q}\right)-M_{q}}{\sqrt{k_{q}^{Y}(x)}}\right),
$$

with $\Phi$ and $\phi$ the c.d.f. and p.d.f. respectively of the standard Gaussian distribution.

Solving the optimization problem in (9) is greatly facilitated by analytical gradients, which are available in our case. Calculations are provided in Appendix C

Remark 1. In the case of the global optimization of black box functions with statistical Bayesian models and in the absence of simulation failures, it is very common to select the observation points as maximizers of the expected improvement. Nevertheless, other ways of selecting the observation points exist, for instance maximizing the improvement probability (see (5) in [38]). In addition, [38] recently showed that the expected improvement strategy and the improvement probability are both special cases of a bi-objective optimization problem that consists in maximizing the conditional expectation (for a maximization problem) and the conditional variance as a function of the observation points.

In future work, it would be interesting to extend the improvement probability and the bi-objective setting to the case of simulation failures, as is done in (9) and (11) for the expected improvement. Our motivation for focusing on the expected improvement is its wide use in the absence of simulation failures and the fact that we obtain the expression (11) which is computationally convenient, in conjunction with Algorithm 1. Furthermore, convergence proofs exist for the optimization algorithm based on the expected improvement [34, 2], which we extend to the simulation failure case in the next section. 


\section{Convergence}

In this section, we prove the convergence of the sequential choice of observation points given by $(9)$, with the slight difference that $(9)$ is replaced by

$$
x_{n+1} \in \underset{x \in \mathcal{D}}{\operatorname{argmax}} \mathbb{E}\left(\max _{\substack{u \in \mathcal{D} \\ \mathbb{P}\left(Z(u)>0 \mid \mathcal{F}_{n, x}\right)=1 \\ \operatorname{var}\left(Y(u) \mid \mathcal{F}_{n, x}\right)=0}} Y(u)-\tilde{M}_{q} \mid \mathcal{F}_{n}\right),
$$

with

$$
\tilde{M}_{q}=\max _{\substack{x \in \mathcal{D} \\ \mathbb{P}\left(Z(x)>0 \mid \mathcal{F}_{n}\right)=1 \\ k_{q}^{Y}(x)=0}} Y(x)
$$

and where $\mathcal{F}_{n, x}$ is the sigma algebra generated by the random variables

$$
I_{1}, I_{1} Y\left(x_{1}\right), \ldots, I_{n}, I_{n} Y\left(x_{n}\right), \mathbf{1}_{Z(x)>0}, \mathbf{1}_{Z(x)>0} Y(x) .
$$

We note that $M_{q}$ corresponds to the maximum over the $q$ observed values of $Y$, while $\tilde{M}_{q}$ is the maximum of $Y$ over the input points $x$ for which it is known (after the $n$ first observations) that $Z(x)>0$ and that $Y(x)=m_{q}^{Y}\left(x, Y_{q}\right)$.

The algorithms given by (9) and (13) coincide when $Z$ and $Y$ are non-degenerate, that is $\left(\xi\left(v_{i}\right)\right)_{i=1, \ldots, r}$ has a non-degenerate distribution for any two-by-two distinct points $v_{1}, \ldots, v_{r} \in$ $\mathcal{D}$, with $\xi=Z$ and $\xi=Y$. These two algorithms can be different when $Y$ or $Z$ are degenerate (which can happen, for instance, when their trajectories are known to satisfy symmetry properties, see e.g. [9]).

Hence, using (13) in the case of degenerate processes enables us to take into account that there are cases where some input points can be known to yield higher values of $Y$ than $\max Y_{q}$ and to yield strictly positive values of $Z$. Furthermore, (13) takes into account the fact that, for $u \notin\left\{x_{1}, \ldots, x_{n}, x\right\}$, the values $\mathbf{1}_{Z(u)>0}$ and $Y(u)$ can have zero uncertainty when $\mathbf{1}_{Z(x)>0}$ and $\mathbf{1}_{Z(x)>0} Y(x)$ are observed.

Following [34, we say that a Gaussian process $\xi$ with continuous trajectories has the no-empty ball (NEB) property if, for any $x_{0} \in \mathcal{D}$ and any $\epsilon>0$,

$$
\inf _{\substack{n \in \mathbb{N} \\ x_{1}, \ldots, x_{n} \in \mathcal{D} \\ x_{i}-x_{0} \| \geq \epsilon, \forall i}} \operatorname{var}\left(\xi\left(x_{0}\right) \mid \xi\left(x_{1}\right), \ldots, \xi\left(x_{n}\right)\right)>0 .
$$

Many standard covariance kernels correspond to Gaussian processes having the NEB property. Indeed, a sufficient condition for the NEB property is that the covariance kernel is stationary with a spectral density decreasing no faster than an inverse polynomial at infinity [34. The most notable covariance function that does not have the NEB property is the squared exponential covariance function [35, but other classical kernel families, such as the Matérn one used in our experiments, do.

We are now in position to state the convergence result.

Theorem 1. Let $\mathcal{D}$ be a compact hypercube of $\mathbb{R}^{d}$. Let $\left(X_{i}\right)_{i \in \mathbb{N}}$ be such that $X_{1}=x_{1}$ is fixed in $\mathcal{D}$ and, for $n \geq 1, X_{n+1}$ is selected by (13). 


\begin{tabular}{l|c|c} 
& $\theta_{Z}=0.1$ & $\theta_{Z}=0.3$ \\
\hline$\theta_{Y}=0.1$ & case 1 & case 3 \\
$\theta_{Y}=0.3$ & case 2 & case 4 \\
\hline
\end{tabular}

Table 1: Studied ranges for the simulations.

1. Assume that $Y$ and $Z$ are Gaussian processes with continuous trajectories. Then, a.s. as $n \rightarrow \infty, \sup _{x \in \mathcal{D}} \mathbb{P}\left(Z(x)>0 \mid \mathcal{F}_{n}\right)\left(m_{q}^{Y}\left(x, Y_{n, s_{n}}\right)-\tilde{M}_{q}\right)^{+} \rightarrow 0$ and $\sup _{x \in \mathcal{D}} \mathbb{P}(Z(x)>$ $\left.0 \mid \mathcal{F}_{n}\right) k_{q}^{Y}(x) \rightarrow 0$.

2. Furthermore, if $Y$ and $Z$ have the $N E B$ property, then $\left(X_{i}\right)_{i \in \mathbb{N}}$ is a.s. dense in $\mathcal{D}$. As a consequence $\max _{i=1, \ldots, n ; Z\left(X_{i}\right)>0} Y\left(X_{i}\right) \rightarrow \max _{u \in \mathcal{D} ; Z(u)>0} Y(u)$ a.s. as $n \rightarrow \infty$.

Proof. Theorem 1 is proved by combining and extending the techniques from [34, 2]. The proof is deferred to Appendix A.

The first part of Theorem 1 states that, as $n \rightarrow \infty$, all the input points $x$ provide an asymptotically negligible expected improvement (similarly, a negligible information). Indeed, they either have a crash probability that goes to one, or a conditional variance that goes to zero and a conditional mean that is no larger than the current maximum $\tilde{M}_{q}$.

The second part of the theorem shows that, as a consequence, the sequence of observation points is dense as $n \rightarrow \infty$ and that the observed maximum converges to the global maximum. The nature of this convergence result is similar to those given in the unconstrained case in [34, 2]. This convergence result guarantees that our suggested algorithm will not leave unexplored regions. Another formulation of Theorem 1 is that our suggested algorithm will not be trapped in local maxima of $Y$.

\section{Simulations on 2D Gaussian processes}

In this section the behavior of our optimization algorithm with crash constraints, which we now call Expected Feasible Improvement with Gaussian Process Classification with signs (EFI GPC sign), is studied on simulated 2D Gaussian processes. We compare this algorithm with the optimization procedure defined in Section 3.2, but where the probabilities of satisfying the constraints are obtained from the classical Gaussian process classifier of [26, 22] based on Expectation Propagation; see Section 2.3. This second algorithm is called EFI GPC EP.

\subsection{Simulations setting}

The two algorithms are run on a function $f:[0,1]^{2} \rightarrow \mathbb{R}$ taken as a realization of a $2 \mathrm{D}$ Gaussian process $Y$. The correlation kernel is a tensorized Matern5_2 kernel with the same correlation length parameter $\theta_{Y}$ in each direction [27]. Observation of $f$ is conditioned on a function $s:[0,1]^{2} \rightarrow\{0,1\}$ such that $s$ is a realization of $\mathbf{1}_{Z>0}$, where $Z$ is a $2 \mathrm{D}$ Gaussian process independent of $Y$. $Z$ is also chosen with a tensorized Matern5_2 kernel with the same parameter $\theta_{Z}$ in each direction.

Two levels of ranges for $\theta_{Y}$ and $\theta_{Z}$ are considered to represent different behaviours of the functions $f$ and $s$. Four cases are studied and summarized in Table 1. In our simulations, the processes $Y$ and $Z$ have mean $\mu^{Y}=\mu^{Z}=0$ and variance $\sigma_{Y}^{2}=\sigma_{Z}^{2}=1$. 

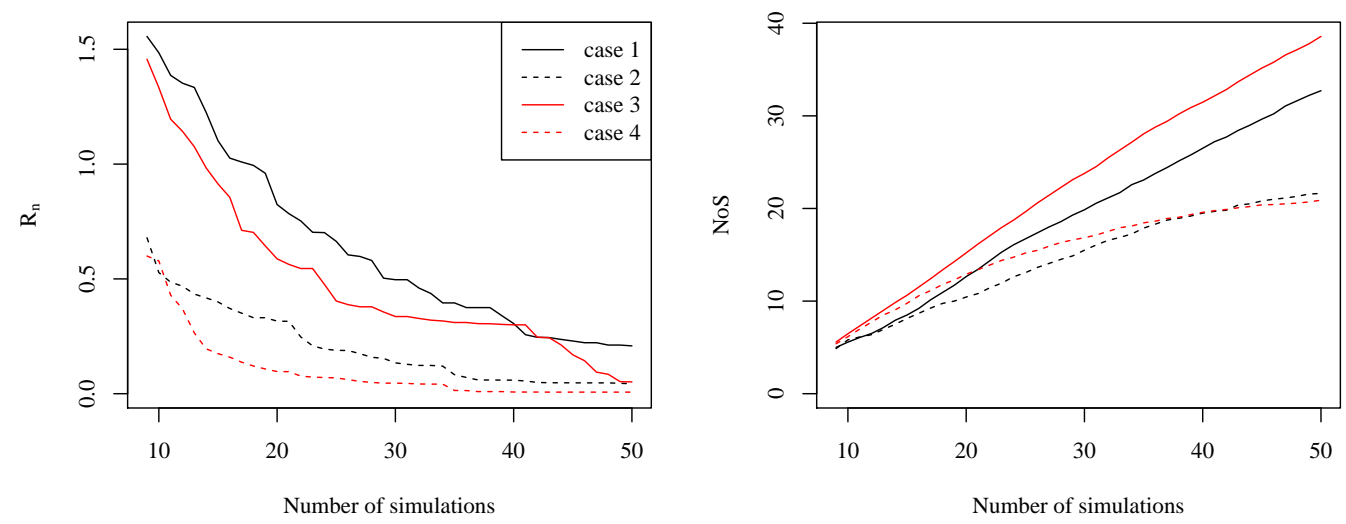

Figure 2: Evolution of average $R_{n}$ (left) and number of successes (NoS, right) along the iteration steps. Four cases of range values are considered (see Table 1). Parameters are estimated by maximum likelihood.

The initial Design of Experiments (DoE) is a maximin Latin hypercube design of 9 points. Then, 41 points are sequentially added according to (15):

$$
x_{n+1} \in \operatorname{argmax}_{x \in \mathcal{D}} \mathrm{P}_{\mathrm{nf}}(x) \times \mathrm{EI}(x) .
$$

Note that, as discussed above, $\mathrm{P}_{\mathrm{nf}}(x)$ is calculated either through our algorithm GPC sign or by a classical GPC, which we denote as GPC EP.

\subsection{Results of our method EFI GPC sign}

In the following we define the regret at step $n$

$$
R_{n}=\max _{x \in[0,1]^{2}, Z(x)>0} Y(x)-\max _{1 \leq i \leq n, Z\left(x_{i}\right)>0} Y\left(x_{i}\right) .
$$

It represents the gap between the global maximum and the current maximum value of the output on the current design of experiments $\left\{x_{1}, \ldots, x_{n}\right\}$. We consider 20 different realizations of $Y$ and $Z$. In Figure 2 (left) the mean of $R_{n}$ is plotted along the iteration steps in the four different cases described in Table 1. It can be noticed that in each case the algorithm converges to the global maximum. The convergence speed depends on the range level. When the correlation length of the process $Y$ is high, i.e. $\theta_{Y}=0.3$, the problem appears to be much easier, independently of the correlation length of $\theta_{Z}$. To a lesser extent, a high range of the process $Z$ also helps to accelerate the convergence.

The evolution of the Number of Successes $(N o S)$ with iteration is plotted in Figure 2 (right). In case 2 and case $4\left(\theta_{Y}=0.3\right)$, the best point is rapidly found, exploration steps are then more numerous and the increase of NoS slows down.

Range parameter estimations for the processes $Y$ and $Z$ are given in the top table of Table 2. The bottom table gives the estimation of trend and variance parameters for both processes. It can be observed that parameter estimation for the process $Z$ is difficult since only signs are available. For instance, $\mu_{Z}$ is overestimated. This reflects under-sampling of crash areas that provide no information on the process $Y$. The situation is different for the process $Y$. Despite failure events, available information and estimation accuracy increase with iterations. 
(a) Range parameters

\begin{tabular}{|l|ccc|ccc|}
\hline & $\theta_{Z}$ & $\hat{\theta}_{Z}$ & $\hat{\theta}_{Z}$ & $\theta_{Y}$ & $\hat{\theta}_{Y}$ & $\hat{\theta}_{Y}$ \\
& true value & iteration 10 & iteration 41 & true value & iteration 10 & iteration 41 \\
\hline Case 1 & 0.1 & $0.44(0.33)$ & $0.37(0.30)$ & 0.1 & $0.17(0.23)$ & $0.10(0.02)$ \\
Case 2 & 0.1 & $0.32(0.29)$ & $0.24(0.25)$ & 0.3 & $0.36(0.23)$ & $0.32(0.16)$ \\
Case 3 & 0.3 & $0.52(0.35)$ & $0.41(0.30)$ & 0.1 & $0.11(0.08)$ & $0.09(0.02)$ \\
Case 4 & 0.3 & $0.49(0.34)$ & $0.51(0.39)$ & 0.3 & $0.34(0.18)$ & $0.28(0.12)$ \\
\hline
\end{tabular}

(b) Trend and variance parameters

\begin{tabular}{|l|cc|cccc|}
\hline & $\hat{\mu}^{Z}$ & $\hat{\mu}^{Z}$ & $\hat{\mu}^{Y}$ & $\hat{\mu}^{Y}$ & $\hat{\sigma}_{Y}^{2}$ & $\hat{\sigma}_{Y}^{2}$ \\
& iteration 10 & iteration 41 & iteration 10 & iteration 41 & iteration 10 & iteration 41 \\
\hline Case 1 & $0.30(0.26)$ & $0.64(0.85)$ & $-0.03(0.57)$ & $-0.09(0.31)$ & $0.68(0.49)$ & $0.82(0.32)$ \\
Case 2 & $0.27(0.33)$ & $0.41(0.39)$ & $0.03(0.70)$ & $0.00(0.53)$ & $0.79(0.51)$ & $0.89(0.70)$ \\
Case 3 & $0.41(0.33)$ & $0.51(0.41)$ & $-0.13(0.36)$ & $-0.06(0.30)$ & $0.66(0.30)$ & $0.83(0.21)$ \\
Case 4 & $0.26(0.20)$ & $0.48(0.41)$ & $-0.16(0.56)$ & $-0.07(0.49)$ & $0.74(0.58)$ & $0.75(0.51)$ \\
\hline
\end{tabular}

Table 2: Method EFI GPC sign at step 10 and 41: (a) Estimation of $\theta_{Z}$ and $\theta_{Y}$, (b) Estimation of $\mu^{Z}$ (true value is 0 ), $\mu^{Y}$ (true value is 0 ) and $\sigma_{Y}^{2}$ (true value is 1 ). Mean (standard deviation) over 20 simulations.

\subsection{Comparison between EFI GPC sign and EFI GPC EP}

The performances of both methods (EFI GPC sign and EFI GPC EP) are compared on the same simulations as previously. It can be seen in Figure D.1 provided in Section D of the supplementary material that the regret of EFI GPC sign converges more rapidly to 0. This can be explained by the fact that the number of sucesses is more important with $E F I$ $G P C$ sign than with EFI GPC EP, since EFI GPC sign avoids crash areas more often (see Figure D.2 from the supplementary material). Parameter estimations of EFI GPC EP are given in Table 3. It can be observed that $Z$-parameter estimation can hardly be compared between methods since the classification models are different. Concerning the process $Y$, the estimated correlation parameters tend towards the real values with more iterations. We note that when the estimated values of $\sigma_{f}^{2}$ are large, the EP classification model is then close to the sign classification model.

An example of the progression of the algorithms in case $1\left(\theta_{Z}=\theta_{Y}=0.1\right)$ is given in Figures D.3 for EFI GPC sign and D.4 for EFI GPC EP in the supplementary material. Both algorithms evolve quite similarly but EFI GPC sign reaches the maximum a bit earlier. Moreover, the number of crashes is lower with EFI GPC sign than with EFI GPC EP.

\section{Industrial case study}

The aim of this section is to find the shape of an automotive fan system that maximizes its efficiency. The geometry of the turbomachinery (more precisely, that of the rotor blades) is described by 15 parameters: 5 chord lengths, 5 stagger angles and 5 heights of maximum camber. A drawing of a blade is provided in Figure E.1 in Section E in the supplementary material. A turbomachinery program has been developed by researchers at the LMFA (Laboratory of acoustics and fluid dynamics) in Ecole Centrale Lyon. It is a multi-physics 1D 
(a) Range parameters

\begin{tabular}{|l|ccc|ccc|}
\hline & $\theta_{Z}$ & $\hat{\theta}_{Z}$ & $\hat{\theta}_{Z}$ & $\theta_{Y}$ & $\hat{\theta}_{Y}$ & $\hat{\theta}_{Y}$ \\
& true value & iteration 10 & iteration 41 & true value & iteration 10 & iteration 41 \\
\hline Case 1 & 0.1 & $0.30(0.35)$ & $0.13(0.10)$ & 0.1 & $0.26(0.48)$ & $0.10(0.03)$ \\
Case 2 & 0.1 & $0.23(0.28)$ & $0.18(0.19)$ & 0.3 & $0.98(1.54)$ & $0.36(0.16)$ \\
Case 3 & 0.3 & $0.47(0.37)$ & $0.34(0.23)$ & 0.1 & $0.21(0.45)$ & $0.12(0.16)$ \\
Case 4 & 0.3 & $0.44(0.35)$ & $0.35(0.29)$ & 0.3 & $0.48(0.73)$ & $0.43(0.73)$ \\
\hline
\end{tabular}

(b) Trend and variance parameters

\begin{tabular}{|l|cc|cccc|}
\hline & $\hat{\sigma}_{f}^{2}$ & $\hat{\sigma}_{f}^{2}$ & $\hat{\mu}^{Y}$ & $\hat{\mu}^{Y}$ & $\hat{\sigma}_{Y}^{2}$ & $\hat{\sigma}_{Y}^{2}$ \\
& iteration 10 & iteration 41 & iteration 10 & iteration 41 & iteration 10 & iteration 41 \\
\hline Case 1 & $8.91(2.77)$ & $10.00(0.00)$ & $-0.10(0.55)$ & $-0.12(0.33)$ & $0.70(0.54)$ & $0.77(0.29)$ \\
Case 2 & $9.79(0.84)$ & $10.00(0.00)$ & $0.04(0.73)$ & $0.14(0.53)$ & $1.03(0.77)$ & $0.94(0.73)$ \\
Case 3 & $9.44(2.07)$ & $10.00(0.00)$ & $-0.11(0.37)$ & $-0.06(0.35)$ & $0.62(0.33)$ & $0.81(0.26)$ \\
Case 4 & $8.37(3.28)$ & $9.47(2.29)$ & $-0.21(0.55)$ & $-0.09(0.56)$ & $1.11(1.81)$ & $0.92(0.84)$ \\
\hline
\end{tabular}

Table 3: Mean and standard deviation (in parentheses) estimates (over 20 runs) of the kernel parameters at two steps of the EFI GPC EP method.

model based on iterative resolution of isentropic efficiency at medium radius, resolution of radial equilibrium, and deduction of blade angles through empirical correlations.

In this context we aim at selecting the geometric parameters that maximize the efficiency of the turbomachinery for a fixed input flow rate and for a fixed pressure rise. The ranges of the 15 geometric parameters are given in Table E.1 in the supplementary material.

For some parameter configurations the simulation does not converge and a NA is returned. These simulation failures can be related to the empirical rules injected in the implementation of the program, which limit its validity domain. Indeed, if the calculation comes out of the admissible domain, the empirical correlations become inaccurate and the simulation is not valid any more.

The issue is to find the optimal geometry considering these failures. A set of initial simulations has been run to explore failure events. We made each geometric parameter vary from its minimum to its maximum around three particular points on the diagonal of the hypercube in dimension 15; Point 1 is close to the minimal corner of the hypercube, Point 2 is at the center and Point 3 is close to the maximal corner of the hypercube. Coordinates are given in Table E.2 in the supplementary material.

The results of these simulations are represented in Figure 3. It can be observed that NAs are more frequent at the edges of the hypercube and near Point1, although no obvious structure can be directly inferred. Besides, highest efficiencies are obtained around the center of the hypercube.

Both methods EFI GPC sign and EFI GPC EP are applied from an initial maximin LHS composed of 75 points. Among them 18 simulations failed. The output range of the valid simulations is roughly [0.3,0.7] and the highest observed efficiency is 0.70 .100 simulations are then successively chosen according to (15). A tensorized matern5_2 kernel is chosen for both the $Z$ and $Y$ processes. As can be seen in Figure 4 , a maximum efficiency of 0.75 is achieved at iteration 22 (resp. 25) for algorithm EFI GPC sign (resp. EFI GPC EP).

Several types of behavior of the algorithms can be observed along the iterations. At the 

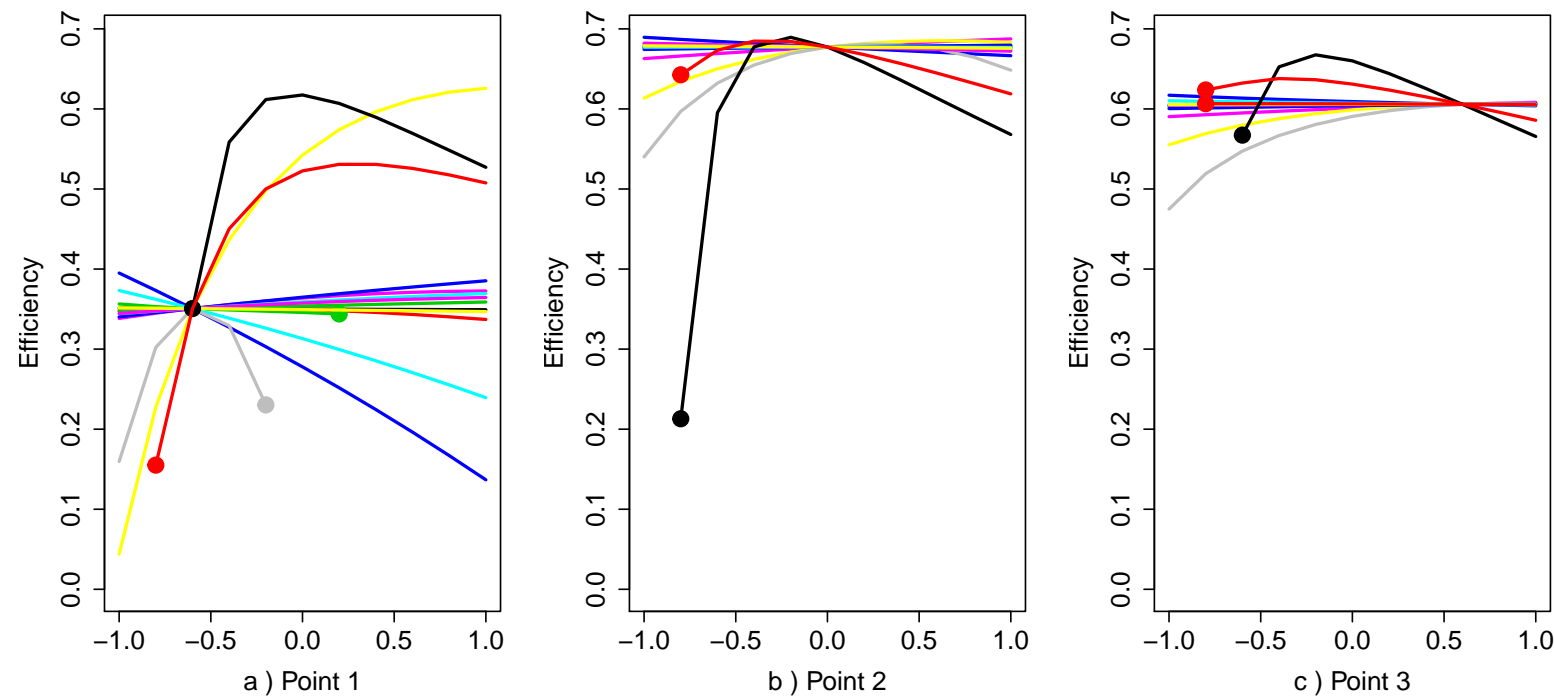

Figure 3: Evolution of the efficiency (output of the code) from min to max in each direction around Point1, Point2 and Point3. The colors indicate the different curves when varying the different input variables. A crash at a given value of $x$ is indicated by the absence of the curve value. The bullets are used to highlight the beginning of the crash ranges for the input variables. To simplify the reading, points are plotted in a normalized domain $[-1,1]^{15}$.

beginning of the algorithms, simulations are added to locally improve efficiency; a single crash occurs over the 20 first points. Then, and especially above iteration 50, the algorithms explore other uncertainty areas and more failures occur. It can be noticed on Figure 4 that our algorithm EFI GPC sign avoids crash areas better than EFI GPC EP. Only 23 failures occur over 100 iterations with EFI GPC sign whereas 34 crashes occur with EFI GPC EP.

\section{Conclusion}

In this paper we have addressed the problem of global optimization of a black-box function under "crash" constraints. To do so, we revisited Gaussian process classification with a model based on observation signs. This model exhibited sharp classification boundaries, which were appropriate in our framework, and allowed us to propose the first algorithm with guaranteed convergence for this problem. Numerical experiments showed promising results, in particular as the algorithm causes fewer simulation failures (in a sense, wasted computational resources) that the current state-of-the-art.

For simplicity, we considered the case where simulations were run one at a time. A possible extension of this work would be to tackle the case of batch-sequential strategies, in the spirit of [8, 36]. We believe that both theoretical and practical aspects could be addressed without major difficulty. Another extension with practical importance would be to tackle problems for which either the objective function and/or the failure events are stochastic; however, a large portion of the proofs proposed here would not apply directly. Finally, convergence rates have not been considered here. Following [5, 32, future work may address this problem. 


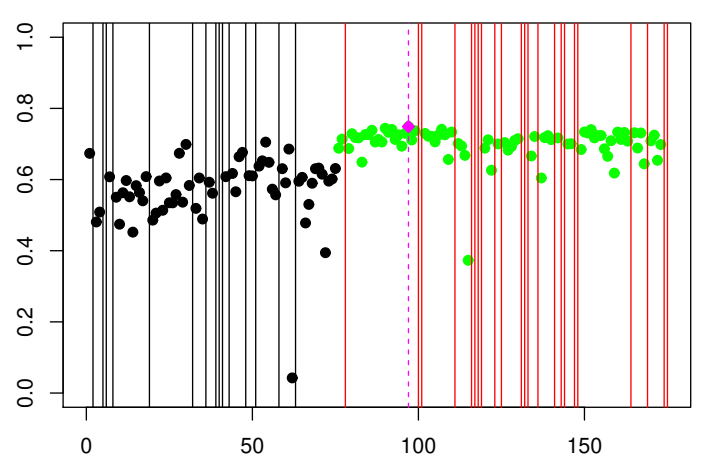

(a) EFI GPC sign

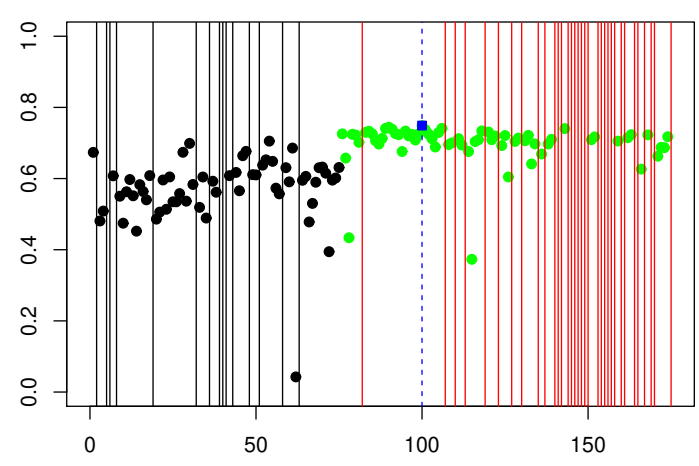

(b) EFI GPCEP

Figure 4: Efficiency values for 175 geometric configurations. The first 75 points come from the initial DoE and are plotted in black. The 100 other points have been added by our optimization algorithms with failures (a) EFI GPC sign and (b) EFI GPC EP. Crashes are represented by vertical lines. On Figure (a) (resp. (b)], for EFI GPC sign (resp. EFI GPC $E P$ ), the best point is represented by a magenta diamond (resp. blue square) and is found at simulation number 97 (resp. 100).

\section{Supplementary material}

The supplementary material contains additional figures and tables for Sections 5 and 6 .

\section{Acknowledgments}

This work was partly funded by the ANR project PEPITO. We are grateful to Jean-Marc Azaïs and David Ginsbourger for discussing the topics in this paper.

\section{A Proofs}

Proof of Lemma 1, With $\phi^{Z_{n}}$ the p.d.f. of $Z_{n}$ and with $s_{n}=\left(i_{1}, \ldots, i_{n}\right)^{\top}$, we have

$$
\begin{aligned}
\mathrm{P}_{\mathrm{nf}}(x)= & \mathbb{P}\left(Z(x)>0 \mid \operatorname{sign}\left(Z_{n}\right)=s_{n}\right) \\
= & \frac{\mathbb{E}\left(\mathbf{1}_{Z(x)>0} \prod_{j=1}^{n} \mathbf{1}_{\operatorname{sign}\left(Z\left(x_{j}\right)\right)=i_{j}}\right)}{\mathbb{P}\left(\operatorname{sign}\left(Z_{n}\right)=s_{n}\right)} \\
= & \frac{\int_{\mathbb{R}^{n}} \phi^{Z_{n}}\left(z_{1}, \ldots, z_{n}\right)\left(\prod_{j=1}^{n} \mathbf{1}_{\operatorname{sign}\left(z_{j}\right)=i_{j}}\right) \mathbb{P}\left(Z(x)>0 \mid Z\left(x_{1}\right)=z_{1}, \ldots, Z\left(x_{n}\right)=z_{n}\right) d z_{1} \ldots d z_{n}}{\mathbb{P}\left(\operatorname{sign}\left(Z_{n}\right)=s_{n}\right)} \\
& =\int_{\mathbb{R}^{n}} \phi_{s_{n}}^{Z_{n}}\left(z_{n}\right) \bar{\Phi}\left(\frac{-m_{n}^{Z}\left(x, z_{n}\right)}{\sqrt{k_{n}^{Z}(x)}}\right) d z_{n} .
\end{aligned}
$$

The equation 16 is obtained by observing that

$$
\frac{\phi^{Z_{n}}\left(z_{1}, \ldots, z_{n}\right)\left(\prod_{j=1}^{n} \mathbf{1}_{\operatorname{sign}\left(z_{j}\right)=i_{j}}\right)}{\mathbb{P}\left(\operatorname{sign}\left(Z_{n}\right)=s_{n}\right)}=\frac{\phi^{Z_{n}}\left(z_{1}, \ldots, z_{n}\right) \mathbf{1}_{\operatorname{sign}\left(Z_{n}\right)=s_{n}}}{\mathbb{P}\left(\operatorname{sign}\left(Z_{n}\right)=s_{n}\right)}=\phi_{s_{n}}^{Z_{n}}\left(z_{n}\right)
$$


by definition of $\phi_{s_{n}}^{Z_{n}}\left(z_{n}\right)$, and that

$$
\mathbb{P}\left(Z(x)>0 \mid Z\left(x_{1}\right)=z_{1}, \ldots, Z\left(x_{n}\right)=z_{n}\right)=\bar{\Phi}\left(\frac{-m_{n}^{Z}\left(x, z_{n}\right)}{\sqrt{k_{n}^{Z}(x)}}\right)
$$

by Gaussian conditioning.

Proof of Lemma 2, For any measurable function $f$, by the law of total expectation and using the independence of $Y,\left(W_{1}, \ldots, W_{n}\right)$ and $Z$, we have

$$
\begin{aligned}
\mathbb{E} & {\left[f\left(I_{1}, \ldots, I_{n}, V_{1}, \ldots, V_{n}\right)\right] } \\
= & \sum_{i_{1}, \ldots, i_{n} \in\{0,1\}} \mathbb{P}_{\mu^{Z}, \theta_{Z}}\left(I_{1}=i_{1}, \ldots, I_{n}=i_{n}\right) \\
& \mathbb{E}\left[f\left(i_{1}, \ldots, i_{n}, i_{1} Y\left(x_{1}\right)+\left(1-i_{1}\right) W_{1}, \ldots, i_{n} Y\left(x_{n}\right)+\left(1-i_{n}\right) W_{n}\right)\right] \\
= & \sum_{i_{1}, \ldots, i_{n} \in\{0,1\}} \mathbb{P}_{\mu^{Z}, \theta_{Z}}\left(I_{1}=i_{1}, \ldots, I_{n}=i_{n}\right) \\
& \int_{\mathbb{R}^{n}} d v \phi_{\mu^{Y}, \theta_{Y}, s_{n}}^{Y}\left(v_{s_{n}}\right)\left(\prod_{\substack{j=1, \ldots, n \\
i_{j}=0}} \phi\left(v_{j}\right)\right) f\left(i_{1}, \ldots, i_{n}, v_{1}, \ldots, v_{n}\right) .
\end{aligned}
$$

This concludes the proof by definition of a p.d.f.

Proof of Lemma 3. Consider measurable functions $f(Y), g(Z), h\left(I_{1}, \ldots, I_{n}\right)$ and $\psi\left(I_{1} Y\left(x_{1}\right), \ldots\right.$, $\left.I_{n} Y\left(x_{n}\right)\right)$. We have, by independence of $Y$ and $Z$,

$$
\begin{aligned}
& \mathbb{E}\left[f(Y) g(Z) h\left(I_{1}, \ldots, I_{n}\right) \psi\left(I_{1} Y\left(x_{1}\right), \ldots, I_{n} Y\left(x_{n}\right)\right)\right] \\
&=\sum_{i_{1}, \ldots, i_{n} \in\{0,1\}} \mathbb{P}\left(I_{1}=i_{1}, \ldots, I_{n}=i_{n}\right) \\
& \mathbb{E}\left[f(Y) g(Z) h\left(i_{1}, \ldots, i_{n}\right) \psi\left(i_{1} Y\left(x_{1}\right), \ldots, i_{n} Y\left(x_{n}\right)\right) \mid I_{1}=i_{1}, \ldots, I_{n}=i_{n}\right] \\
&=\sum_{i_{1}, \ldots, i_{n} \in\{0,1\}} \mathbb{P}\left(I_{1}=i_{1}, \ldots, I_{n}=i_{n}\right) h\left(i_{1}, \ldots, i_{n}\right) \\
& \mathbb{E}\left[f(Y) \psi\left(i_{1} Y\left(x_{1}\right), \ldots, i_{n} Y\left(x_{n}\right)\right)\right] \mathbb{E}\left[g(Z) \mid I_{1}=i_{1}, \ldots, I_{n}=i_{n}\right] \\
&= \sum_{i_{1}, \ldots, i_{n} \in\{0,1\}} \mathbb{P}\left(I_{1}=i_{1}, \ldots, I_{n}=i_{n}\right) h\left(i_{1}, \ldots, i_{n}\right) \\
& \mathbb{E}\left[\psi\left(i_{1} Y\left(x_{1}\right), \ldots, i_{n} Y\left(x_{n}\right)\right) \mathbb{E}\left[f(Y) \mid Y_{n, s_{n}}\right]\right] \mathbb{E}\left[g(Z) \mid I_{1}=i_{1}, \ldots, I_{n}=i_{n}\right] .
\end{aligned}
$$

The last display can be written as, with $\mathcal{L}_{n}$ the distribution of

$$
\begin{array}{r}
I_{1}, \ldots, I_{n}, I_{1} Y\left(x_{1}\right), \ldots, I_{n} Y\left(x_{n}\right), \\
\int_{\mathbb{R}^{2 n}} d \mathcal{L}_{n}\left(i_{1}, \ldots, i_{n}, i_{1} y_{1}, \ldots, i_{n} y_{n}\right) h\left(i_{1}, \ldots, i_{n}\right) \psi\left(i_{1} y_{1}, \ldots, i_{n} y_{n}\right) \\
\mathbb{E}\left[f(Y) \mid Y_{n, s_{n}}=Y_{q}\right] \mathbb{E}\left[g(Z) \mid I_{1}=i_{1}, \ldots, I_{n}=i_{n}\right],
\end{array}
$$

where $Y_{q}$ is as defined in the statement of the lemma. This concludes the proof. 
We now address the proof of Theorem 1. We let $\left(X_{i}\right)_{i \in \mathbb{N}}$ be the random observation points, such that $X_{i}$ is obtained from (13) and (14) for $i \in \mathbb{N}$. The next lemma shows that conditioning on the random observation points and observed values works "as if" the observation points $X_{1}, \ldots, X_{n}$ were non-random.

Lemma 4. For any $x_{1}, \ldots, x_{k} \in \mathcal{D}, i_{1}, \ldots, i_{k} \in\{0,1\}^{k}$ and $i_{1} y_{1}, \ldots, i_{k} y_{k} \in \mathbb{R}^{k}$, the conditional distribution of $(Y, Z)$ given

$$
\begin{aligned}
& X_{1}=x_{1}, \operatorname{sign}\left(Z\left(X_{1}\right)\right)=i_{1}, \operatorname{sign}\left(Z\left(X_{1}\right)\right) Y\left(X_{1}\right)=i_{1} y_{1}, \ldots, \\
& X_{k}=x_{k}, \operatorname{sign}\left(Z\left(X_{k}\right)\right)=i_{k}, \operatorname{sign}\left(Z\left(X_{k}\right)\right) Y\left(X_{k}\right)=i_{k} y_{k}
\end{aligned}
$$

is the same as the conditional distribution of $(Y, Z)$ given

$$
\operatorname{sign}\left(Z\left(x_{1}\right)\right)=i_{1}, \operatorname{sign}\left(Z\left(x_{1}\right)\right) Y\left(x_{1}\right)=i_{1} y_{1}, \ldots, \operatorname{sign}\left(Z\left(x_{k}\right)\right)=i_{k}, \operatorname{sign}\left(Z\left(x_{k}\right)\right) Y\left(x_{k}\right)=i_{k} y_{k} .
$$

Proof. This lemma can be shown similarly as Proposition 2.6 in [2].

Proof of Theorem 1. For $k \in \mathbb{N}$, we remark that $\mathcal{F}_{k}$ is the sigma-algebra generated by

$$
X_{1}, \operatorname{sign}\left(Z\left(X_{1}\right)\right), \operatorname{sign}\left(Z\left(X_{1}\right)\right) Y\left(X_{1}\right), \ldots, X_{k}, \operatorname{sign}\left(Z\left(X_{k}\right)\right), \operatorname{sign}\left(Z\left(X_{k}\right)\right) Y\left(X_{k}\right) .
$$

We let $\mathbb{E}_{k}, \mathbb{P}_{k}$ and $\operatorname{var}_{k}$ denote the expectation, probability and variance conditionally on $\mathcal{F}_{k}$. For $x \in \mathcal{D}$, we let $\mathbb{E}_{k, x}, \mathbb{P}_{k, x}$ and $\operatorname{var}_{k, x}$ denote the expectation, probability and variance conditionally on

$X_{1}, \operatorname{sign}\left(Z\left(X_{1}\right)\right), \operatorname{sign}\left(Z\left(X_{1}\right)\right) Y\left(x_{1}\right), \ldots, X_{k}, \operatorname{sign}\left(Z\left(X_{k}\right)\right), \operatorname{sign}\left(Z\left(X_{k}\right)\right) Y\left(X_{k}\right), x, \operatorname{sign}(Z(x)), \operatorname{sign}(Z(x)) Y(x)$.

We let $\sigma_{k}^{2}(u)=\operatorname{var}_{k}(Y(u)), m_{k}(u)=\mathbb{E}_{k}[Y(u)]$ and $P_{k}(u)=\mathbb{P}_{k}(Z(u)>0)$. We also let $\sigma_{k, x}^{2}(u)=\operatorname{var}_{k, x}(Y(u)), m_{k, x}(u)=\mathbb{E}_{k, x}[Y(u)]$ and $P_{k, x}(u)=\mathbb{P}_{k, x}(Z(u)>0)$.

With these notations, the observation points satisfy, for $k \in \mathbb{N}$,

$$
X_{k+1} \in \operatorname{argmax}_{x \in \mathcal{D}} \mathbb{E}_{k}\left(\max _{\substack{u: P_{k, x}(u)=1 \\ \sigma_{k, x}(u)=0}} Y(u)-M_{k}\right),
$$

where

$$
M_{k}=\max _{\substack{u: P_{k}(u)=1 \\ \sigma_{k}(u)=0}} Y(u) .
$$

We first show that (17) can be defined as a stepwise uncertainty reduction (SUR) sequential design [2]. We have

$$
\begin{aligned}
& X_{k+1} \in \operatorname{argmax}_{x \in \mathcal{D}} \mathbb{E}_{k}\left(\max _{\begin{array}{c}
P_{k, x}(u)=1 \\
\sigma_{k, x}(u)=0
\end{array}} Y(u)-\max _{\begin{array}{l}
P_{k}(u)=1 \\
\sigma_{k}(u)=0
\end{array}} Y(u)\right) \\
& \in \operatorname{argmin}_{x \in \mathcal{D}} \mathbb{E}_{k}\left(\mathbb{E}_{k, x}\left(\max _{Z(u)>0} Y(u)\right)-\max _{\begin{array}{c}
P_{k, x}(u)=1 \\
\sigma_{k, x}(u)=0
\end{array}} Y(u)\right)
\end{aligned}
$$


since the second term in 18 does not depend on $x$ and from the law of total expectation. We let

$$
H_{k}=\mathbb{E}_{k}\left(\max _{Z(u)>0} Y(u)-\max _{\begin{array}{c}
P_{k}(u)=1 \\
\sigma_{k}(u)=0
\end{array}} Y(u)\right)
$$

and

$$
H_{k, x}=\mathbb{E}_{k, x}\left(\max _{Z(u)>0} Y(u)-\max _{\substack{P_{k, x}(u)=1 \\ \sigma_{k, x}(u)=0}} Y(u)\right) .
$$

Then we have for $k \geq 1$

$$
X_{k+1} \in \operatorname{argmin}_{x \in \mathcal{D}} \mathbb{E}_{k}\left(H_{k, x}\right) .
$$

We have, using the law of total expectation, and since $\mathbb{E}_{k, x}\left[\max _{P_{k, x}(u)=1, \sigma_{k, x}(u)=0} Y(u)\right]=$ $\max _{P_{k, x}(u)=1, \sigma_{k, x}(u)=0} Y(u)$,

$$
\begin{aligned}
& H_{k}-\mathbb{E}_{k}\left(H_{k+1}\right)=\mathbb{E}_{k}\left(\max _{\substack{P_{k, X_{k+1}}(u)=1 \\
\sigma_{k, X_{k+1}}(u)=0}} Y(u)-\max _{\begin{array}{c}
P_{k}(u)=1 \\
\sigma_{k}(u)=0
\end{array}} Y(u)\right) \\
& \geq 0
\end{aligned}
$$

since, for all $u, x \in \mathcal{D}, \sigma_{k, x}(u) \leq \sigma_{k}(u)$ and $P_{k}(u)=1$ implies $P_{k, x}(u)=1$. Hence $\left(H_{k}\right)_{k \in \mathbb{N}}$ is a supermartingale and of course $H_{k} \geq 0$ for all $k \in \mathbb{N}$. Also $\left|H_{1}\right| \leq 2 \mathbb{E}_{1}\left[\max _{u \in \mathcal{D}}|Y(u)|\right]$ so that $H_{1}$ is bounded in $L^{1}$, since the mean value of the maximum of a continuous Gaussian process on a compact set is finite. Hence, from Theorem 6.23 in [14], $H_{k}$ converges a.s. as $k \rightarrow \infty$ to a finite random variable. Hence, as in the proof of Theorem 3.10 in [2], we have $H_{k}-\mathbb{E}_{k}\left(H_{k+1}\right)$ goes to 0 a.s. as $k \rightarrow \infty$. Hence, by definition of $X_{k+1}$ we obtain $\sup _{x \in \mathcal{D}}\left(H_{k}-\mathbb{E}_{k}\left(H_{k, x}\right)\right) \rightarrow 0$ a.s. as $k \rightarrow \infty$. This yields, from the law of total expectation,

$$
\begin{aligned}
& 0 \longleftarrow{ }_{n \rightarrow \infty}^{a . s .} \sup _{x \in \mathcal{D}} \mathbb{E}_{k}\left(\max _{\begin{array}{l}
\max _{k, x}(u)=1 \\
\sigma_{k, x}(u)=0
\end{array}} Y(u)-\max _{\begin{array}{c}
P_{k}(u)=1 \\
\sigma_{k}(u)=0
\end{array}} Y(u)\right) \\
& \geq \sup _{x \in \mathcal{D}} \mathbb{E}_{k}\left[\mathbf{1}_{Z(x)>0}\left(Y(x)-M_{k}\right)^{+}\right] \\
& \geq \sup _{x \in \mathcal{D}} P_{k}(x) \gamma\left(m_{k}(x)-M_{k}, \sigma_{k}(x)\right),
\end{aligned}
$$

from Lemma 3 and $(12)$, where

$$
\gamma(a, b)=a \Phi\left(\frac{a}{b}\right)+b \phi\left(\frac{a}{b}\right) .
$$

Recall from Section 3 in [34] that $\gamma$ is continuous and satisfies $\gamma(a, b)>0$ if $b>0$ and $\gamma(a, b) \geq a$ if $a>0$. We have for $k \in \mathbb{N}, 0 \leq \sigma_{k}(u) \leq \max _{v \in \mathcal{D}} \sqrt{\operatorname{var}(Y(v))}<\infty$. Also, with the same proof as that of Proposition 2.9 in [2], we can show that the sequence of random functions $\left(m_{k}\right)_{k \in \mathbb{N}}$ converges a.s. uniformly on $\mathcal{D}$ to a continuous random function $m_{\infty}$ on $\mathcal{D}$. Thus, from (19), by compacity, we have, a.s. as $k \rightarrow \infty, \sup _{x \in \mathcal{D}} P_{k}(x)\left(m_{k}(x)-M_{k}\right)^{+} \rightarrow 0$ and $\sup _{x \in \mathcal{D}} P_{k}(x) \sigma_{k}(x) \rightarrow 0$. Hence, Part 1. is proved. 
Let us address Part 2. For all $\tau \in \mathbb{N}$, consider fixed $v_{1}, \ldots, v_{N_{\tau}} \in \mathcal{D}$ for which $\max _{u \in \mathcal{D}}$ $\min _{i=1, \ldots, N_{\tau}}\left\|u-v_{i}\right\| \leq 1 / \tau$. Consider the event $E_{\tau}=\left\{\exists u \in \mathcal{D} ; \inf _{i \in \mathbb{N}}\left\|X_{i}-u\right\| \geq 2 / \tau\right\}$. Then, $E_{\tau}$ implies the event $E_{v, \tau}=\cup_{j=1}^{N_{\tau}} E_{v, \tau, j}$ where $E_{v, \tau, j}=\left\{\inf _{i \in \mathbb{N}}\left\|X_{i}-v_{j}\right\| \geq 1 / \tau\right\}$. Let us now show that $\mathbb{P}\left(E_{v, \tau, j}\right)=0$ for $j=1, \ldots, N_{\tau}$. Assume that $E_{v, \tau, j} \cap \mathcal{C}$ holds, where $\mathcal{C}$ is the event in Part 1. of the theorem, with $\mathbb{P}(\mathcal{C})=1$. Since $Y$ has the NEB property, we have $\liminf \operatorname{in}_{k \rightarrow \infty} \sigma_{k}\left(v_{j}\right)>0$. Hence, $P_{k}\left(v_{j}\right) \rightarrow 0$ as $k \rightarrow \infty$ since $\mathcal{C}$ is assumed. We then have

$$
\operatorname{var}\left(\mathbf{1}_{Z\left(v_{j}\right)>0} \mid \mathbf{1}_{Z\left(X_{1}\right)>0}, \ldots, \mathbf{1}_{Z\left(X_{k}\right)>0}\right)=P_{k}\left(v_{j}\right)\left(1-P_{k}\left(v_{j}\right)\right) \rightarrow 0
$$

a.s. as $k \rightarrow \infty$. But we have

$$
\begin{aligned}
& \operatorname{var}\left(\mathbf{1}_{Z\left(v_{j}\right)>0} \mid \mathbf{1}_{Z\left(X_{1}\right)>0}, \ldots, \mathbf{1}_{Z\left(X_{k}\right)>0}\right) \\
& =\mathbb{E}\left[\left(\mathbf{1}_{Z\left(v_{j}\right)>0}-P_{k}\left(v_{j}\right)\right)^{2} \mid \mathbf{1}_{Z\left(X_{1}\right)>0}, \ldots, \mathbf{1}_{Z\left(X_{k}\right)>0}\right] \\
& =\mathbb{E}\left[\mathbb{E}\left[\left(\mathbf{1}_{Z\left(v_{j}\right)>0}-P_{k}\left(v_{j}\right)\right)^{2} \mid Z\left(x_{1}\right), \ldots, Z\left(x_{k}\right)\right] \mid \mathbf{1}_{Z\left(X_{1}\right)>0}, \ldots, \mathbf{1}_{Z\left(X_{k}\right)>0}\right] .
\end{aligned}
$$

Since $P_{k}\left(v_{j}\right)$ is a function of $Z\left(x_{1}\right), \ldots, Z\left(x_{n}\right)$, we obtain

$$
\begin{aligned}
& \operatorname{var}\left(\mathbf{1}_{Z\left(v_{j}\right)>0} \mid \mathbf{1}_{Z\left(X_{1}\right)>0}, \ldots, \mathbf{1}_{Z\left(X_{k}\right)>0}\right) \\
& \geq \mathbb{E}\left[\operatorname{var}\left(\mathbf{1}_{Z\left(v_{j}\right)>0} \mid Z\left(x_{1}\right), \ldots, Z\left(x_{k}\right)\right) \mid \mathbf{1}_{Z\left(X_{1}\right)>0}, \ldots, \mathbf{1}_{Z\left(X_{k}\right)>0}\right] \\
& =\mathbb{E}\left[g\left(\bar{\Phi}\left(\frac{-m_{k}\left(v_{j}\right)}{\sigma_{k}\left(v_{j}\right)}\right)\right) \mid \mathbf{1}_{Z\left(X_{1}\right)>0}, \ldots, \mathbf{1}_{Z\left(X_{k}\right)>0}\right],
\end{aligned}
$$

with $g(t)=t(1-t)$ and with $\bar{\Phi}$ as in Lemma 1. We let $S=\sup _{k \in \mathbb{N}}\left|m_{k}\left(v_{j}\right)\right|$ and $s=$ $\inf _{k \in \mathbb{N}} \sigma_{k}\left(v_{j}\right)$. Then, from the uniform convergence of $m_{k}$ discussed below and from the NEB property of $Z$, we have $\mathbb{P}\left(E_{S, s}\right)=1$ where $E_{S, s}=\{S<+\infty, s>0\}$. Then, if $E_{v, \tau, j} \cap \mathcal{C} \cap E_{S, s}$ holds, we have

$$
\begin{aligned}
& \operatorname{var}\left(\mathbf{1}_{Z\left(v_{j}\right)>0} \mid \mathbf{1}_{Z\left(X_{1}\right)>0}, \ldots, \mathbf{1}_{Z\left(X_{k}\right)>0}\right) \\
& \geq \mathbb{E}\left[g\left(\bar{\Phi}\left(\frac{S}{s}\right)\right) \mid \mathbf{1}_{Z\left(X_{1}\right)>0}, \ldots, \mathbf{1}_{Z\left(X_{k}\right)>0}\right] \\
& \quad \rightarrow \rightarrow_{k \rightarrow \infty}^{\text {a.s. }} \mathbb{E}\left[g\left(\bar{\Phi}\left(\frac{S}{s}\right)\right) \mid \mathcal{F}_{Z, \infty}\right],
\end{aligned}
$$

where $\mathcal{F}_{Z, \infty}=\sigma\left(\left\{\mathbf{1}_{\left.Z\left(X_{i}\right)>0\right)}\right\}_{i \in \mathbb{N}}\right)$ from Theorem 6.23 in [14]. Almost surely, conditionally on $\mathcal{F}_{Z, \infty}$ we have a.s. $S<\infty$ and $s>0$. Hence we obtain that, on the event $E_{v, \tau, j} \cap \mathcal{A}$ with $\mathbb{P}(\mathcal{A})=1, \operatorname{var}\left(\mathbf{1}_{Z\left(v_{j}\right)>0} \mid \mathbf{1}_{Z\left(X_{1}\right)>0}, \ldots, \mathbf{1}_{Z\left(X_{k}\right)>0}\right)$ does not go to zero. Hence, from (20), we have $\mathbb{P}\left(E_{v, \tau, j}\right)=0$. This yields that $\left(X_{i}\right)_{i \in \mathbb{N}}$ is a.s. dense in $\mathcal{D}$. Hence, since $\{u ; Z(u)>0\}$ is an open set, we have $\max _{i ; Z\left(X_{i}\right)>0} Y\left(X_{i}\right) \rightarrow \max _{Z(u)>0} Y(u)$ a.s. as $n \rightarrow \infty$.

\section{B Stochastic approximation of the likelihood gradient for Gaus- sian process based classification}

In Appendixes $\mathrm{B}$ and $\mathrm{C}$, for two matrices $A$ and $B$ of sizes $a \times d$ and $b \times d$, and for a function $h: \mathbb{R}^{d} \times \mathbb{R}^{d} \rightarrow \mathbb{R}$, let $h(A, B)$ be the $a \times b$ matrix $\left[h\left(a_{i}, b_{j}\right)\right]_{i=1, \ldots, a, j=1, \ldots, b}$, where $a_{i}$ and $b_{j}$ are the lines $i$ and $j$ of $A$ and $B$. 
Let $s_{n}=\left(i_{1}, \ldots, i_{n}\right) \in\{0,1\}^{n}$ be fixed. Assume that the likelihood $\mathbb{P}_{\mu, \theta}\left(\operatorname{sign}\left(Z_{n}\right)=s_{n}\right)$ has been evaluated by $\hat{\mathbb{P}}_{\mu, \theta}\left(\operatorname{sign}\left(Z_{n}\right)=s_{n}\right)$. Assume also that realizations $z_{n}^{(1)}, \ldots, z_{n}^{(N)}$, approximately following the conditional distribution of $Z_{n}$ given $\operatorname{sign}\left(Z_{n}\right)=s_{n}$, are available.

Let $\mathcal{Z}=\left\{z_{n} \in \mathbb{R}^{n}: \operatorname{sign}\left(z_{n}\right)=s_{n}\right\}$. Treating $x_{1}, \ldots, x_{n}$ as $d$-dimensional line vectors, let $\mathbf{x}$ be the matrix $\left(x_{1}^{\top}, \ldots, x_{n}^{\top}\right)^{\top}$. Then we have

$$
\mathbb{P}_{\mu, \theta}\left(\operatorname{sign}\left(Z_{n}\right)=s_{n}\right)=\int_{\mathcal{Z}} \frac{1}{(2 \pi)^{n / 2}} \frac{1}{\sqrt{\left|k_{\theta}^{Z}(\mathbf{x}, \mathbf{x})\right|}} e^{\frac{-1}{2}\left(z_{n}-\mu 1_{n}\right)^{\top} k_{\theta}^{Z}(\mathbf{x}, \mathbf{x})^{-1}\left(z_{n}-\mu 1_{n}\right)} d z_{n},
$$

where $1_{n}=(1, \ldots, 1)^{\top} \in \mathbb{R}^{n}$ and $|$.$| denotes the determinant.$

Derivating with respect to $\mu$ yields

$$
\begin{aligned}
\frac{\partial}{\partial \mu} \mathbb{P}_{\mu, \theta}\left(\operatorname{sign}\left(Z_{n}\right)=s_{n}\right)= & \int_{\mathcal{Z}} \frac{1}{(2 \pi)^{n / 2}} \frac{1}{\sqrt{\left|k_{\theta}^{Z}(\mathbf{x}, \mathbf{x})\right|}} e^{\frac{-1}{2}\left(z_{n}-\mu 1_{n}\right)^{\top} k_{\theta}^{Z}(\mathbf{x}, \mathbf{x})^{-1}\left(z_{n}-\mu 1_{n}\right)} \\
& \left(1_{n}^{\top} k_{\theta}^{Z}(\mathbf{x}, \mathbf{x})^{-1}\left(z_{n}-\mu 1_{n}\right)\right) d z_{n} \\
= & \left.\mathbb{P}_{\mu, \theta}\left(\operatorname{sign}\left(Z_{n}\right)=s_{n}\right) \mathbb{E}_{\mu, \theta}\left(1_{n}^{\top} k_{\theta}^{Z}(\mathbf{x}, \mathbf{x})^{-1}\left(Z_{n}-\mu 1_{n}\right) \mid \operatorname{sign}\left(Z_{n}\right)=s_{n}\right)\right),
\end{aligned}
$$

where $\mathbb{E}_{\mu, \theta}$ means that the conditional expectation is calculated under the assumption that $Z$ has constant mean function $\mu$ and covariance function $k_{\theta}^{Z}$. Hence we have the stochastic approximation $\hat{\nabla}_{\mu}$ for $\partial / \partial \mu \mathbb{P}_{\mu, \theta}\left(\operatorname{sign}\left(Z_{n}\right)=s_{n}\right)$ given by

$$
\hat{\nabla}_{\mu}=\hat{\mathbb{P}}_{\mu, \theta}\left(\operatorname{sign}\left(Z_{n}\right)=s_{n}\right) \frac{1}{N} \sum_{i=1}^{N} 1_{n}^{\top} k_{\theta}^{Z}(\mathbf{x}, \mathbf{x})^{-1}\left(z_{n}^{(i)}-\mu 1_{n}\right) .
$$

Derivating with respect to $\theta_{i}$ for $i=1, \ldots, p$ yields, with $\operatorname{adj}(M)$ the adjugate of a matrix $M$,

$$
\begin{aligned}
& \frac{\partial}{\partial \theta_{i}} \mathbb{P}_{\mu, \theta}\left(\operatorname{sign}\left(Z_{n}\right)=s_{n}\right)= \\
& \int_{\mathcal{Z}}\left(\frac{-1}{2}\left|k_{\theta}^{Z}(\mathbf{x}, \mathbf{x})\right|^{-1} \operatorname{Tr}\left(\operatorname{adj}\left(k_{\theta}^{Z}(\mathbf{x}, \mathbf{x})\right) \frac{\partial k_{\theta}^{Z}(\mathbf{x}, \mathbf{x})}{\partial \theta_{i}}\right)\right. \\
& \left.+\frac{1}{2}\left(z_{n}-\mu 1_{n}\right)^{\top} \frac{\partial k_{\theta}^{Z}(\mathbf{x}, \mathbf{x})}{\partial \theta_{i}} k_{\theta}^{Z}(\mathbf{x}, \mathbf{x})^{-1} \frac{\partial k_{\theta}^{Z}(\mathbf{x}, \mathbf{x})}{\partial \theta_{i}}\left(z_{n}-\mu 1_{n}\right)\right) \\
& \quad \frac{1}{(2 \pi)^{n / 2}} \frac{1}{\sqrt{\left|k_{\theta}^{Z}(\mathbf{x}, \mathbf{x})\right|}} e^{\frac{-1}{2}\left(z_{n}-\mu 1_{n}\right)^{\top} k_{\theta}^{Z}(\mathbf{x}, \mathbf{x})^{-1}\left(z_{n}-\mu 1_{n}\right)} d z_{n} \\
& =\mathbb{P}_{\mu, \theta}\left(\operatorname{sign}\left(Z_{n}\right)=s_{n}\right) \\
& \mathbb{E}_{\mu, \theta}\left(\frac{-1}{2}\left|k_{\theta}^{Z}(\mathbf{x}, \mathbf{x})\right|^{-1} \operatorname{Tr}\left(\operatorname{adj}\left(k_{\theta}^{Z}(\mathbf{x}, \mathbf{x})\right) \frac{\partial k_{\theta}^{Z}(\mathbf{x}, \mathbf{x})}{\partial \theta_{i}}\right)\right. \\
& \left.\left.+\frac{1}{2}\left(Z_{n}-\mu 1_{n}\right)^{\top} \frac{\partial k_{\theta}^{Z}(\mathbf{x}, \mathbf{x})}{\partial \theta_{i}} k_{\theta}^{Z}(\mathbf{x}, \mathbf{x})^{-1} \frac{\partial k_{\theta}^{Z}(\mathbf{x}, \mathbf{x})}{\partial \theta_{i}}\left(Z_{n}-\mu 1_{n}\right) \mid \operatorname{sign}\left(Z_{n}\right)=s_{n}\right)\right) .
\end{aligned}
$$

Hence we have the stochastic approximation $\hat{\nabla}_{\theta_{i}}$ for $\partial / \partial \theta_{i} \mathbb{P}_{\mu, \theta}\left(\operatorname{sign}\left(Z_{n}\right)=s_{n}\right)$ given by

$$
\begin{aligned}
& \hat{\nabla}_{\theta_{i}}=\hat{\mathbb{P}}_{\mu, \theta}\left(\operatorname{sign}\left(Z_{n}\right)=s_{n}\right) \frac{1}{N} \sum_{i=1}^{N}\left(\frac{-1}{2}\left|k_{\theta}^{Z}(\mathbf{x}, \mathbf{x})\right|^{-1} \operatorname{Tr}\left(\operatorname{adj}\left(k_{\theta}^{Z}(\mathbf{x}, \mathbf{x})\right) \frac{\partial k_{\theta}^{Z}(\mathbf{x}, \mathbf{x})}{\partial \theta_{i}}\right)\right. \\
& \left.+\frac{1}{2}\left(z_{n}^{(i)}-\mu 1_{n}\right)^{\top} \frac{\partial k_{\theta}^{Z}(\mathbf{x}, \mathbf{x})}{\partial \theta_{i}} k_{\theta}^{Z}(\mathbf{x}, \mathbf{x})^{-1} \frac{\partial k_{\theta}^{Z}(\mathbf{x}, \mathbf{x})}{\partial \theta_{i}}\left(z_{n}^{(i)}-\mu 1_{n}\right)\right) .
\end{aligned}
$$


Remark 2. Several implementations of algorithms are available to obtain the realizations $z_{n}^{(1)}, \ldots, z_{n}^{(N)}$, as discussed after Algorithm 1. It may also be the case that some implementations provide both the estimate $\hat{\mathbb{P}}_{\mu, \theta}\left(\operatorname{sign}\left(Z_{n}\right)=s_{n}\right)$ and the realizations $z_{n}^{(1)}, \ldots, z_{n}^{(N)}$.

\section{Expressions of the mean and covariance of the conditional Gaussian process and of the gradient of the acquisition function}

Let $\mu^{Y}$ and $k^{Y}$ be the mean and covariance functions of $Y$. Treating $x_{1}, \ldots, x_{n}$ as $d$ dimensional line vectors, let $\mathbf{x}_{q}$ be the matrix extracted from $\left(x_{1}^{\top}, \ldots, x_{n}^{\top}\right)^{\top}$ by keeping only the lines which indices $j$ satisfy $i_{j}=1$.

We first recall the classical expressions of GP conditioning:

$$
\begin{aligned}
m_{q}^{Y}\left(x, Y_{q}\right) & =\mu^{Y}+k^{Y}\left(x, \mathbf{x}_{q}\right)\left(k^{Y}\left(\mathbf{x}_{q}, \mathbf{x}_{q}\right)\right)^{-1}\left(Y_{q}-\mu^{Y}\right) \\
k_{q}^{Y}\left(x, x^{\prime}\right) & =k^{Y}\left(x, x^{\prime}\right)-k^{Y}\left(x, \mathbf{x}_{q}\right)\left(k^{Y}\left(\mathbf{x}_{q}, \mathbf{x}_{q}\right)\right)^{-1} k^{Y}\left(\mathbf{x}_{q}, x^{\prime}\right) .
\end{aligned}
$$

$\nabla_{x} m_{q}^{Y}\left(x, Y_{q}\right)$ and $\nabla_{x} k_{q}^{Y}(x, x)$ are straightforward provided that $\nabla_{x} k^{Y}(x, y)$ is available:

$$
\begin{aligned}
\nabla_{x} m_{q}^{Y}\left(x, Y_{q}\right) & =\left[\nabla_{x} k^{Y}\left(x, \mathbf{x}_{q}\right)\right]\left(k^{Y}\left(\mathbf{x}_{q}, \mathbf{x}_{q}\right)\right)^{-1}\left(Y_{q}-\mu^{Y}\right) \\
\nabla_{x} k_{q}^{Y}(x, x) & =\nabla_{x} k^{Y}(x, x)-2 k^{Y}\left(x, \mathbf{x}_{q}\right)\left(k^{Y}\left(\mathbf{x}_{q}, \mathbf{x}_{q}\right)\right)^{-1} \nabla_{x} k^{Y}\left(\mathbf{x}_{q}, x\right) .
\end{aligned}
$$

Then:

$\nabla_{x} E I_{q}(x)=\Phi\left(\frac{m_{q}^{Y}\left(x, Y_{q}\right)-M_{q}}{\sqrt{k_{q}^{Y}(x, x)}}\right) \nabla_{x} m_{q}^{Y}\left(x, Y_{q}\right)+\phi\left(\frac{M_{q}-m_{q}^{Y}\left(x, Y_{q}\right)}{\sqrt{k_{q}^{Y}(x, x)}}\right) \frac{1}{2 \sqrt{k_{q}^{Y}(x, x)}} \nabla_{x} k_{q}^{Y}(x, x)$.

For $\mathrm{P}_{\mathrm{nf}}(x)$, using the approximation of Algorithm 1, we have:

$$
\widehat{\mathrm{P}_{\mathrm{nf}}}(x)=\frac{1}{N} \sum_{i=1}^{N} \bar{\Phi}\left(\frac{-m_{n}^{Z}\left(x, z_{n}^{(i)}\right)}{\sqrt{k_{n}^{Z}(x, x)}}\right)
$$

with $k_{n}^{Z}(x, x)$ as $k_{n}^{Y}(x, x)$ and

$$
m_{n}^{Z}\left(x, z_{n}^{(i)}\right)=\mu^{Z}+k^{Z}(x, \mathbf{x})\left(k^{Z}(\mathbf{x}, \mathbf{x})\right)^{-1}\left(z_{n}^{(i)}-\mu^{Z}\right) .
$$

Applying the standard differentiation rules delivers:

$$
\nabla_{x} \widehat{\mathrm{P}_{\mathrm{nf}}}(x)=\frac{1}{N} \sum_{i=1}^{N} \phi\left(\frac{m_{n}^{Z}\left(x, z_{n}^{(i)}\right)}{\sqrt{k_{n}^{Z}(x, x)}}\right)\left[\frac{1}{\sqrt{k_{n}^{Z}(x, x)}} \nabla_{x} m_{n}^{Z}\left(x, z_{n}^{(i)}\right)-\frac{m_{n}^{Z}\left(x, z_{n}^{(i)}\right)}{2\left[k_{n}^{Z}(x, x)\right]^{3 / 2}} \nabla_{x} k_{n}^{Z}(x, x)\right] .
$$

The gradient of the acquisition function can then be obtained using the product rule. 


\section{References}

[1] D. Azzimonti and D. Ginsbourger. Estimating orthant probabilities of high-dimensional Gaussian vectors with an application to set estimation. Journal of Computational and Graphical Statistics, 27(2):255-267, 2018.

[2] J. Bect, F. Bachoc, and D. Ginsbourger. A supermartingale approach to Gaussian process based sequential design of experiments. Bernoulli, 25(4A):2883-2919, 2019.

[3] R. Benassi, J. Bect, and E. Vazquez. Robust Gaussian process-based global optimization using a fully Bayesian expected improvement criterion. In International Conference on Learning and Intelligent Optimization, pages 176-190. Springer, 2011.

[4] Z. I. Botev. The normal law under linear restrictions: simulation and estimation via minimax tilting. Journal of the Royal Statistical Society: Series B (Statistical Methodology), 79(1):125-148, 2017.

[5] A. D. Bull. Convergence rates of efficient global optimization algorithms. Journal of Machine Learning Research, 12(Oct):2879-2904, 2011.

[6] M. A. Gelbart, J. Snoek, and R. P. Adams. Bayesian optimization with unknown constraints. In UAI, 2014.

[7] A. Genz. Numerical computation of multivariate normal probabilities. Journal of computational and graphical statistics, 1(2):141-149, 1992.

[8] D. Ginsbourger, R. Le Riche, and L. Carraro. Kriging is well-suited to parallelize optimization. In Computational intelligence in expensive optimization problems, pages 131162. Springer, 2010.

[9] D. Ginsbourger, O. Roustant, and N. Durrande. On degeneracy and invariances of random fields paths with applications in Gaussian process modelling. Journal of statistical planning and inference, 170:117-128, 2016.

[10] R. Gramacy and H. Lee. Optimization under unknown constraints. Bayesian Statistics, 9, 2011.

[11] R. B. Gramacy, G. A. Gray, S. Le Digabel, H. K. Lee, P. Ranjan, G. Wells, and S. M. Wild. Modeling an augmented Lagrangian for blackbox constrained optimization. Technometrics, 58(1):1-11, 2016.

[12] J. M. Hernandez-Lobato, M. Gelbart, M. Hoffman, R. Adams, and Z. Ghahramani. Predictive entropy search for Bayesian optimization with unknown constraints. In International Conference on Machine Learning, pages 1699-1707, 2015.

[13] D. Jones, M. Schonlau, and W. Welch. Efficient global optimization of expensive black box functions. Journal of Global Optimization, 13:455-492, 1998.

[14] O. Kallenberg. Foundations of Modern Probability. Second edition. Springer-Verlag, 2002. 
[15] K. Kandasamy, W. Neiswanger, J. Schneider, B. Poczos, and E. P. Xing. Neural architecture search with Bayesian optimisation and optimal transport. In Advances in Neural Information Processing Systems, pages 2016-2025, 2018.

[16] A. Keane and P. Nair. Computational approaches for aerospace design: the pursuit of excellence. John Wiley \& Sons, 2005.

[17] D. V. Lindberg and H. K. Lee. Optimization under constraints by applying an asymmetric entropy measure. Journal of Computational and Graphical Statistics, 24(2):379-393, 2015.

[18] A. F. López-Lopera, F. Bachoc, N. Durrande, and O. Roustant. Finite-dimensional Gaussian approximation with linear inequality constraints. SIAM/ASA Journal on Uncertainty Quantification, 6(3):1224-1255, 2018.

[19] H. Maatouk and X. Bay. A New Rejection Sampling Method for Truncated Multivariate Gaussian Random Variables Restricted to Convex Sets, pages 521-530. Springer International Publishing, Cham, 2016.

[20] S. P. Meyn and R. L. Tweedie. Markov chains and stochastic stability. Springer Science \& Business Media, 2012.

[21] J. B. Mockus, V. Tiesis, and A. Žilinskas. The application of Bayesian methods for seeking the extremum. In L. C. W. Dixon and G. P. Szegö, editors, Towards Global Optimization, volume 2, pages 117-129, North Holland, New York, 1978.

[22] H. Nickisch and C. E. Rasmussen. Approximations for binary Gaussian process classification. Journal of Machine Learning Research, 9(Oct):2035-2078, 2008.

[23] A. Pakman and L. Paninski. Exact Hamiltonian Monte Carlo for truncated multivariate Gaussians. Journal of Computational and Graphical Statistics, 23(2):518-542, 2014.

[24] V. Picheny. A stepwise uncertainty reduction approach to constrained global optimization. In Artificial Intelligence and Statistics, pages 787-795, 2014.

[25] V. Picheny, R. B. Gramacy, S. Wild, and S. Le Digabel. Bayesian optimization under mixed constraints with a slack-variable augmented Lagrangian. In Advances in Neural Information Processing Systems, pages 1435-1443, 2016.

[26] C. Rasmussen and C. Williams. Gaussian Processes for Machine Learning. The MIT Press, Cambridge, 2006.

[27] O. Roustant, D. Ginsbourger, and Y. Deville. DiceKriging, DiceOptim: Two R packages for the analysis of computer experiments by Kriging-based metamodeling and optimization. Journal of statistical software, 51(1):1-55, 2012.

[28] M. Sacher, R. Duvigneau, O. Le Maitre, M. Durand, E. Berrini, F. Hauville, and J.-A. Astolfi. A classification approach to efficient global optimization in presence of noncomputable domains. Structural and Multidisciplinary Optimization, 58(4):1537-1557, 2018. 
[29] M. J. Sasena, P. Papalambros, and P. Goovaerts. Exploration of metamodeling sampling criteria for constrained global optimization. Engineering optimization, 34(3):263-278, 2002.

[30] M. Schonlau, W. J. Welch, and D. R. Jones. Global versus local search in constrained optimization of computer models. Lecture Notes-Monograph Series, pages 11-25, 1998.

[31] J. Snoek, H. Larochelle, and R. P. Adams. Practical Bayesian optimization of machine learning algorithms. In Advances in neural information processing systems, pages 2951$2959,2012$.

[32] N. Srinivas, A. Krause, S. Kakade, and M. Seeger. Gaussian process optimization in the bandit setting: no regret and experimental design. In Proceedings of the 27th International Conference on Machine Learning, pages 1015-1022, 2010.

[33] J. Taylor and Y. Benjamini. RestrictedMVN: multivariate normal restricted by affine constraints. https://cran.r-project.org/web/packages/restrictedMVN/index.html, 2017. [Online; 02-Feb-2017].

[34] E. Vazquez and J. Bect. Convergence properties of the expected improvement algorithm with fixed mean and covariance functions. Journal of Statistical Planning and inference, 140(11):3088-3095, 2010.

[35] E. Vazquez and J. Bect. Pointwise consistency of the kriging predictor with known mean and covariance functions. In mODa 9-Advances in Model-Oriented Design and Analysis, pages 221-228. Springer, 2010.

[36] J. Wu and P. Frazier. The parallel knowledge gradient method for batch Bayesian optimization. In Advances in Neural Information Processing Systems, pages 3126-3134, 2016.

[37] A. Zhigljavsky and A. Žilinskas. Selection of a covariance function for a Gaussian random field aimed for modeling global optimization problems. Optimization Letters, 13(2):249$259,2019$.

[38] A. Žilinskas and J. Calvin. Bi-objective decision making in global optimization based on statistical models. Journal of Global Optimization, 74(4):599-609, 2019. 